\title{
K.U.Leuven
}

\section{On the sequence of consecutive powers of a matrix in a Boolean algebra*}

\author{
B. De Schutter and B. De Moor
}

If you want to cite this report, please use the following reference instead:

B. De Schutter and B. De Moor, "On the sequence of consecutive powers of a matrix in a Boolean algebra," SIAM Journal on Matrix Analysis and Applications, vol. 21, no. 1, pp. 328-354, 1999. 


\title{
ON THE SEQUENCE OF CONSECUTIVE POWERS OF A MATRIX IN A BOOLEAN ALGEBRA
}

\author{
BART DE SCHUTTER* AND BART DE MOOR ${ }^{\dagger}$
}

\begin{abstract}
In this paper we consider the sequence of consecutive powers of a matrix in a Boolean algebra. We characterize the ultimate behavior of this sequence, we study the transient part of the sequence and we derive upper bounds for the length of this transient part. We also indicate how these results can be used in the analysis of Markov chains and in max-plus-algebraic system theory for discrete event systems.

Key words. Boolean algebra, Boolean matrices, transient behavior, Markov chains, max-plus
\end{abstract} algebra

AMS subject classifications. 06E99, 15A99, 16Y99

1. Introduction. In this paper we consider the sequence of consecutive powers of a matrix in a Boolean algebra. This sequence reaches a "cyclic" behavior after a finite number of terms. Even for more complex algebraic structures, such as the max-plus algebra (which has maximization and addition as its basic operations) this ultimate behavior has already been studied extensively by several authors (See, e.g., $[1,9,13,26]$ and the references therein). In this paper we completely characterize the ultimate behavior of the sequence of the consecutive powers of a matrix in a Boolean algebra. Furthermore, we also study the transient part of this sequence. More specifically, we give upper bounds for the length of the transient part of the sequence as a function of structural parameters of the matrix.

Our main motivation for studying this problem lies in the max-plus-algebraic system theory for discrete event systems. Furthermore, our results can also be used in the analysis of the transient behavior of Markov chains.

This paper is organized as follows. In $\S 2$ we introduce some of the notations and concepts from number theory, Boolean algebra, matrix algebra and graph theory that will be used in the paper. In $\S 3$ we characterize the ultimate behavior of the sequence of consecutive powers of a given matrix in a Boolean algebra, and we derive upper bounds for the length of the transient part of this sequence. In $\S 4$ we briefly sketch how our results can be used in the analysis of Markov chains and in the max-plusalgebraic system theory for discrete event systems. In this section we also explain why we have restricted ourselves to Boolean algebras in this paper and we indicate some of the phenomena that should be taken into account when extending our results to more general algebraic structures. Finally we present some conclusions in $\S 5$.

\section{Preliminaries.}

2.1. Notation, definitions and some lemmas from number theory. In this paper we use "vector" as a synonym for "column matrix". If $a$ is a vector, then $a_{i}$ is the $i$ th component of $a$. If $A$ is a matrix, then $a_{i j}$ or $(A)_{i j}$ is the entry on the $i$ th row and the $j$ th column, and $A_{\alpha \beta}$ is the submatrix of $A$ obtained by removing all rows that are not indexed by the set $\alpha$ and all columns that are not indexed by the set $\beta$.

\footnotetext{
${ }^{*}$ Control Laboratory, Faculty of Information Technology and Systems, Delft University of Technology, P.O. Box 5031, 2600 GA Delft, The Netherlands (b.deschutter@its.tudelft.nl).

${ }^{\dagger}$ ESAT/SISTA, K.U.Leuven, Kardinaal Mercierlaan 94, B-3001 Heverlee (Leuven), Belgium (bart.demoor@esat.kuleuven.ac.be).
} 
TABLE 2.1

The operations $\oplus$ and $\otimes$ for the Boolean algebra $(\{\mathbf{O}, \mathbf{1}\}, \oplus, \otimes)$.

\begin{tabular}{|c||c|c|}
\hline$\oplus$ & 0 & 1 \\
\hline \hline 0 & 0 & 1 \\
\hline 1 & 1 & 1 \\
\hline
\end{tabular}

\begin{tabular}{|c||c|c|}
\hline$\otimes$ & 0 & 1 \\
\hline \hline 0 & 0 & 0 \\
\hline 1 & 0 & 1 \\
\hline
\end{tabular}

The set of the real numbers is denoted by $\mathbb{R}$, the set of the nonnegative integers by $\mathbb{N}$, and the set of the positive integers by $\mathbb{N}_{0}$.

If $S$ is a set, then the number of elements of $S$ is denoted by $\# S$. If $\gamma$ is a set of positive integers then the least common multiple of the elements of $\gamma$ is denoted by lcm $\gamma$ and the greatest common divisor of the elements of $\gamma$ is denoted by gcd $\gamma$.

If $x \in \mathbb{R}$ then $\lceil x\rceil$ is the smallest integer that is larger than or equal to $x$, and $\lfloor x\rfloor$ is the largest integer that is less than or equal to $x$.

LEMmA 2.1. Let $p, q \in \mathbb{N}_{0}$ be coprime. The smallest integer $n$ such that for any integer $m \geq n$, there exist two nonnegative integers $\alpha$ and $\beta$ such that $m=\alpha p+\beta q$, is given by $n=(p-1)(q-1)$.

Proof. See, e.g., the proof of Lemma 3.5.5 of [5].

Let $a_{1}, a_{2}, \ldots, a_{n} \in \mathbb{N}_{0}$ with $\operatorname{gcd}\left(a_{1}, a_{2}, \ldots, a_{n}\right)=1$. We define $g\left(a_{1}, a_{2}, \ldots, a_{n}\right)$ to be the largest positive integer $N$ for which the equation $a_{1} x_{1}+a_{2} x_{2}+\ldots+a_{n} x_{n}=N$ subject to $x_{1}, x_{2}, \ldots, x_{n} \in \mathbb{N}$, has no solution. From Lemma 2.1 it follows that $g(a, b)=(a-1)(b-1)-1=a b-a-b$. Although a formula exists for the case where $n=3$, no general formulas are known for $n \geq 4$. However, some upper bounds have been proved $[4,11]$ :

LEMmA 2.2. If $a_{1}, a_{2}, \ldots, a_{n} \in \mathbb{N}_{0}$ with $a_{1}<a_{2}<\ldots<a_{n}$ and $\operatorname{gcd}\left(a_{1}, \ldots, a_{n}\right)=$ 1 , then $g\left(a_{1}, \ldots, a_{n}\right) \leq\left(a_{1}-1\right)\left(a_{n}-1\right)-1$.

LEMMA 2.3. If $a_{1}, a_{2}, \ldots, a_{n} \in \mathbb{N}_{0}$ with $a_{1}<a_{2}<\ldots<a_{n}$ and $\operatorname{gcd}\left(a_{1}, \ldots, a_{n}\right)=$ 1 , then we have $g\left(a_{1}, \ldots, a_{n}\right) \leq 2 a_{n-1}\left\lfloor\frac{a_{n}}{n}\right\rfloor-a_{n}$.

2.2. Boolean algebra. A Boolean algebra is an algebraic structure of the form $(\mathbb{B}, \oplus, \otimes)$ with $\mathbb{B}=\{\boldsymbol{O}, \mathbf{1}\}$ such that the operations $\oplus$ and $\otimes$ applied on $\mathcal{O}$ and $\mathbf{1}$ yield the results of Table 2.1 , where $\oplus$ and $\otimes$ are associative, and where $\otimes$ is distributive with respect to $\oplus$. The element $\mathcal{O}$ is called the Boolean zero element, $\mathbf{l}$ is called the Boolean identity element, $\oplus$ is called the Boolean addition and $\otimes$ is called the Boolean multiplication.

Some examples of Boolean algebras are: (\{false, true $\}$, or, and $),(\{0, \infty\}, \min ,+)$, $(\{0,1\}, \max , \cdot),(\{\emptyset, \mathbb{N}\}, \cup, \cap),(\{0,1\}, \max , \min ),(\{-\infty, \infty\}, \max , \min )$, and so on (see $[1,15])$. In this paper we shall use the following examples of Boolean algebra in order to transform known results from max-plus algebra and from nonnegative matrix algebra to Boolean algebra:

1. The Boolean algebra $(\{-\infty, 0\}, \max ,+)$ is a subalgebra of the max-plus algebra $(\mathbb{R} \cup\{-\infty\}$, max, + ).

2. The Boolean algebra $(\{0, \mathfrak{p}\},+, \cdot)$ where $\mathfrak{p}$ stands for an arbitrary positive number ${ }^{1}$ can be considered as a Boolean restriction of nonnegative algebra.

A matrix with entries in $\mathbb{B}$ is called a Boolean matrix. The operations $\oplus$ and $\otimes$ are extended to matrices as follows. If $A, B \in \mathbb{B}^{m \times n}$ then we have

$$
(A \oplus B)_{i j}=a_{i j} \oplus b_{i j}
$$

\footnotetext{
${ }^{1}$ So $\mathfrak{p}+\mathfrak{p}=\mathfrak{p}$ and $\mathfrak{p} \cdot \mathfrak{p}=\mathfrak{p}$.
} 
for all $i, j$. If $A \in \mathbb{B}^{m \times p}$ and $B \in \mathbb{B}^{p \times n}$ then

$$
(A \otimes B)_{i j}=\bigoplus_{k=1}^{p} a_{i k} \otimes b_{k j}
$$

for all $i, j$. Note that these definitions resemble the definitions of the sum and the product of matrices in linear algebra but with $\oplus$ instead of + and $\otimes$ instead of .

The $n$ by $n$ Boolean identity matrix is denoted by $\mathcal{I}_{n}$, the $m$ by $n$ Boolean zero matrix is denoted by $\mathcal{O}_{m \times n}$, and the $m$ by $n$ matrix all the entries of which are equal to 1 is denoted by $\mathcal{E}_{m \times n}$. If the dimensions of these matrices are not indicated they should be clear from the context.

The Boolean matrix power of the matrix $A \in \mathbb{B}^{n \times n}$ is defined as follows:

$$
A^{\otimes^{0}}=\mathcal{I}_{n}, \quad \text { and } \quad A^{\otimes^{k}}=A \otimes A^{\otimes^{k-1}} \quad \text { for } k=1,2, \ldots
$$

If we permute the rows or the columns of the Boolean identity matrix, we obtain a Boolean permutation matrix. If $P \in \mathbb{B}^{n \times n}$ is a Boolean permutation matrix, then we have $P \otimes P^{T}=P^{T} \otimes P=\mathcal{I}_{n}$. A matrix $R \in \mathbb{B}^{m \times n}$ is a Boolean upper triangular matrix if $r_{i j}=\mathrm{O}$ for all $i, j$ with $i>j$.

2.3. Boolean algebra and graph theory. We assume that the reader is familiar with basic concepts of graph theory such as directed graph, path, (elementary) circuit, and so on (see, e.g., $[1,18,27])$. In this paper we shall use the definitions of [1] since they are well suited for our proofs. Sometimes these definitions differ slightly from the definitions adopted by other schools in the literature. The most important differences are:

- In this paper we also consider empty paths, i.e., paths that consist of only one vertex and have length 0 . However, unless it is explicitly specified, we always assume that paths have a nonzero length.

- The precedence graph of the matrix $A \in \mathbb{B}^{n \times n}$, by denoted by $\mathcal{G}(A)$, is a directed graph with vertices $1,2, \ldots, n$ and an arc $j \rightarrow i$ for each $a_{i j} \neq \mathcal{O}$. Note that vertex $i$ is the end point of this arc.

- A directed graph is called strongly connected if for any two different vertices $v_{i}, v_{j}$ there exists a path from $v_{i}$ to $v_{j}$. Note that this implies that a graph consisting of one vertex (with or without a loop) is always strongly connected.

- A matrix is irreducible if its precedence graph is strongly connected. Since according to the definition we use a graph with only one vertex is always strongly connected, the 1 by 1 Boolean zero matrix [o] is irreducible. However, the 1 by 1 Boolean zero matrix [0] is the only Boolean zero matrix that is irreducible.

Let us now give a graph-theoretic interpretation of the Boolean matrix power. Let $A \in \mathbb{B}^{n \times n}$ and let $k \in \mathbb{N}_{0}$. Recall that there is an arc $j \rightarrow i$ in $\mathcal{G}(A)$ if and only if $a_{i j}=1$. Since

$$
\left(A^{\otimes}\right)_{i j}=\bigoplus_{i_{1}, i_{2}, \ldots, i_{k-1}} a_{i i_{1}} \otimes a_{i_{1} i_{2}} \otimes \ldots \otimes a_{i_{k-1} j}
$$

for all $i, j$ and since $\mathcal{O}$ is absorbing for $\otimes,\left(A^{\otimes^{k}}\right)_{i j}$ is equal to $\mathbf{l}$ if and only if there exists a path of length $k$ from vertex $j$ to vertex $i$ in $\mathcal{G}(A)$.

A maximal strongly connected subgraph (m.s.c.s.) $\mathcal{G}_{\text {sub }}$ of a directed graph $\mathcal{G}$ is a strongly connected subgraph that is maximal, i.e., if we add an extra vertex (and some extra arcs) of $\mathcal{G}$ to $\mathcal{G}_{\text {sub }}$ then $\mathcal{G}_{\text {sub }}$ is no longer strongly connected. 
A well-known result from matrix algebra states that any square matrix can be transformed into a block upper diagonal matrix with irreducible blocks by simultaneously reordering the rows and columns of the matrix (see, e.g., [1, 2, 5, 12, 17, 22] for the proof of this theorem and for its interpretation in terms of graph theory and Markov chains):

TheOREM 2.4. If $A \in \mathbb{B}^{n \times n}$ then there exists a permutation matrix $P \in \mathbb{B}^{n \times n}$ such that the matrix $\hat{A}=P \otimes A \otimes P^{T}$ is a block upper triangular matrix of the form

$$
\hat{A}=\left[\begin{array}{cccc}
\hat{A}_{11} & \hat{A}_{12} & \ldots & \hat{A}_{1 l} \\
\mathcal{O} & \hat{A}_{22} & \ldots & \hat{A}_{2 l} \\
\vdots & \vdots & \ddots & \vdots \\
\mathcal{O} & \mathcal{O} & \ldots & \hat{A}_{l l}
\end{array}\right]
$$

with $l \geq 1$ and where the matrices $\hat{A}_{11}, \hat{A}_{22}, \ldots, \hat{A}_{l l}$ are square and irreducible. The matrices $\hat{A}_{11}, \hat{A}_{22}, \ldots, \hat{A}_{l l}$ are uniquely determined to within simultaneous permutation of their rows and columns, but their ordering in (1) is not necessarily unique.

The form in (1) is called the Frobenius normal form of the matrix $A$. If $A$ is irreducible then there is only one block in (1) and then $A$ is a Frobenius normal form of itself. Each diagonal block of $\hat{A}$ corresponds to an m.s.c.s. of the precedence graph of $\hat{A}$.

TheOREm 2.5. If $A \in \mathbb{B}^{n \times n}$ is irreducible, then

$$
\exists k_{0} \in \mathbb{N}, \exists c \in \mathbb{N}_{0}, \text { such that } \forall k \geq k_{0}: A^{\otimes^{k+c}}=\lambda^{\otimes^{c}} \otimes A^{\otimes^{k}}
$$

where $\lambda$ is equal to 1 if there exists a circuit in $\mathcal{G}(A)$, and equal to 0 otherwise.

Proof. See, e.g., $[1,7,13]$.

The smallest $c$ for which (2) holds is called the cyclicity [1], index of cyclicity [2] or index of $i_{\text {mprimitivity }}^{2}[5,12]$ of the matrix $A$. The cyclicity $c(A)$ of a matrix $A$ is equal to the cyclicity of the precedence graph $\mathcal{G}(A)$ of $A$ and can be computed as follows. The cyclicity of a strongly connected graph or of an m.s.c.s. is the greatest common divisor of the lengths of all the circuits of the given graph or m.s.c.s. If an m.s.c.s. or a graph contains no circuits then its cyclicity is equal to 0 by definition. The cyclicity of general graph is the least common multiple of the nonzero cyclicities of the m.s.c.s.'s of the given graph.

Lemma 2.6. If $A \in \mathbb{B}^{n \times n}$ is irreducible then $c(A) \leq n$.

Proof. Let $c=c(A)$. Since $A$ is irreducible, $\mathcal{G}(A)$ contains only one m.s.c.s. If $A=[\mathrm{o}]$ then we have $c=0 \leq 1=n$.

From now on we assume that $\bar{A} \neq \mathcal{O}$. Since $c$ is the greatest common divisor of the lengths of the (elementary) circuits in $\mathcal{G}(A), c$ is maximal if there is only one circuit and if this circuit has length $n$. In that case we have $c=n$. In the other cases, $c$ will be less than $n$.

So $c(A) \leq n$.

LEMMA 2.7. Let $A \in \mathbb{B}^{n \times n}$ be irreducible and let $c$ be the cyclicity of $A$. Consider $i, j \in\{1,2, \ldots, n\}$. If $c>0$ and if there exists a (non-empty) path of length $l_{1}$ from $j$ to $i$ and a (non-empty) path of length $l_{2}$ from $j$ to $i$ then there exists a (possibly negative) integer $z$ such that $l_{2}=l_{1}+z c$.

\footnotetext{
${ }^{2}$ We prefer to use the word "cyclicity" or "index of cyclicity" in this paper in order to avoid confusion with the concept "index of primitivity" $[2,25]$ of a nonnegative matrix $A$, which is defined to be the least positive integer $\gamma(A)$ such that all the entries of $A^{\gamma(A)}$ are positive.
} 
Proof. This lemma is a reformulation of Lemma 3.4 .1 of [5] that states that if $\mathcal{G}$ is a strongly connected directed graph with cyclicity $c$ then for each pair of vertices $j$ and $i$ of $\mathcal{G}$, the lengths of the paths from $j$ to $i$ are congruent modulo $c$.

Remark 2.8. Consider $A \in \mathbb{B}^{n \times n}$ and $i, j \in\{1,2, \ldots, n\}$. Let $l_{i j}$ be the length of the shortest path from vertex $j$ to vertex $i$ in $\mathcal{G}(A)$. Note that Lemma 2.7 does not imply that there exists a path of length $l_{i j}+k c$ from $j$ to $i$ for every $k \in \mathbb{N}$. $\diamond$ In the next section we discuss upper bounds for the integer $k_{0}$ that appears in Theorem 2.5. We also extend this theorem to Boolean matrices that are not irreducible.

3. Consecutive powers of a Boolean matrix. In this section we consider the sequence $\left\{A^{\otimes^{k}}\right\}_{k=1}^{\infty}$ where $A$ is a Boolean matrix. First we consider matrices with a cyclicity that is equal to 0 . Next we consider matrices with a cyclicity that is larger than or equal to 1 . Here we shall make a distinction between four different cases depending on whether the given matrix is irreducible or not, and on whether its cyclicity is equal to 1 , or larger than or equal to 1 . Of course the last case that will be considered is the most general one, but for the other cases we can provide tighter upper bounds on the length of the transient part of the sequence $\left\{A^{\otimes^{k}}\right\}_{k=1}^{\infty}$ and that is why we consider four different cases.

If possible we also give examples of matrices for which the sequence of the consecutive matrix powers exhibits the longest possible transient behavior.

3.1. Boolean matrices with a cyclicity that is equal to 0. LEMMA 3.1. Let $A \in \mathbb{B}^{n \times n}$. If $c(A)=0$ then we have $A^{\otimes^{k}}=\mathcal{O}_{n \times n}$ for all $k \geq n$.

Proof. If the cyclicity of $A$ is equal to 0 , then there are no circuits in $\mathcal{G}(A)$, which means that there do not exist paths in $\mathcal{G}(A)$ with a length that is larger than or equal to $n$ since in such paths at least one vertex would appear twice, which implies that such paths contain a circuit. Therefore, we have $A^{\otimes^{k}}=\mathcal{O}$ for all $k \geq n$.

ExAmple 3.2. If there exists a permutation matrix $P$ such that $A \in \mathbb{B}^{n \times n}$ can be written as

$$
\hat{A}=P \otimes A \otimes P^{T}=\left[\begin{array}{ccccc}
0 & 1 & 0 & \ldots & 0 \\
0 & 0 & 1 & \ldots & 0 \\
\vdots & \vdots & \vdots & \ddots & \vdots \\
0 & 0 & 0 & \ldots & 1 \\
0 & 0 & 0 & \ldots & 0
\end{array}\right]
$$

then the upper bound of Lemma 3.1 is tight, i.e., we have $A^{\otimes^{k}} \neq \mathcal{O}$ for $k=$ $1,2, \ldots, n-1$ and $A^{\otimes^{k}}=\mathcal{O}$ for all $k \geq n$. The graph of the matrix $\hat{A}$ is represented in Figure 3.1. Note that $c(A)=c(\hat{A})=0$ since $\mathcal{G}(\hat{A})$ contains no circuits and since the transformation from $A$ to $\hat{A}$ corresponds to a simultaneous reordering of the rows and the columns of $A$ (or of the vertices of $\mathcal{G}(A)$ ).

From now on we only consider matrices with a cyclicity that is larger than or equal to 1 .

3.2. Boolean matrices with cyclicity 1. Theorem 3.3. Let $A \in \mathbb{B}^{n \times n}$. If the cyclicity of $A$ is equal to 1 and if $A$ is irreducible, then we have $A^{\otimes^{k+1}}=A^{\otimes^{k}}=$ $\mathcal{E}_{n \times n}$ for all $k \geq(n-1)^{2}+1$.

Proof. This theorem can be considered as the Boolean equivalent of Theorem 4.14 of [2] or of Theorem 3.5.6 of [5]. Note that $A$ cannot be equal to [0] since $c(A)=1$. 


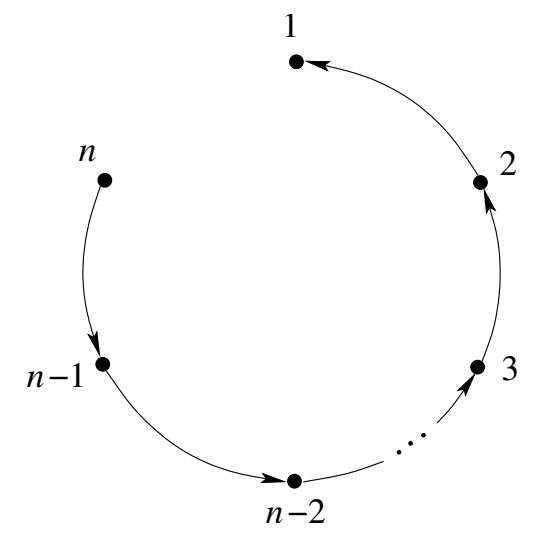

FIG. 3.1. The precedence graph of the matrix $\hat{A}$ of Example 3.2.

If more information about the structure of $A$ is known (such as the number of diagonal entries that are equal to $\mathbf{1}$, the length of the shortest elementary circuit of $\mathcal{G}(A)$, or whether $A$ is symmetrically nonnegative) other upper bounds for the length of the transient part of the sequence $\left\{A^{\otimes^{k}}\right\}_{k=1}^{\infty}$ where $A$ is a Boolean matrix with cyclicity 1 can be found in $\S 2.4$ of [2].

EXAMPLE 3.4. If there exists a permutation matrix $P$ such $A \in \mathbb{B}^{n \times n}$ can be written as

$$
\hat{A}=P \otimes A \otimes P^{T}=\left[\begin{array}{ccccc}
0 & 1 & 0 & \ldots & 0 \\
0 & 0 & 1 & \ldots & 0 \\
\vdots & \vdots & 0 & \ddots & \vdots \\
1 & 0 & 0 & \ldots & 1 \\
1 & 0 & 0 & \ldots & 0
\end{array}\right]
$$

then the bound in Theorem 3.3 is tight: we have $A^{\otimes^{k}}=\mathcal{E}$ for all $k \geq(n-1)^{2}+1$ but $A^{\otimes}{ }^{(n-1)^{2}} \neq 1$. Let us now show that the latter part of this statement indeed holds. Since the transformation from $A$ to $\hat{A}=P \otimes A \otimes P^{T}$ corresponds to a simultaneous reordering of the rows and the columns of $A$, we may assume without loss of generality that $P$ is the identity matrix. So $A=\hat{A}$. If $n=2$ then we have $(n-1)^{2}+1=2$. Since

$$
A=\left[\begin{array}{ll}
1 & 1 \\
1 & 0
\end{array}\right] \text { and } A^{\otimes^{2}}=A^{\otimes^{3}}=\ldots=\mathcal{E}
$$

we indeed have $A^{\otimes^{(n-1)^{2}}} \neq \mathcal{E}$ if $n=2$.

From now on we assume that $n>2$. In Figure 3.2 we have drawn $\mathcal{G}(A)$. There are two elementary circuits in $\mathcal{G}(A)$ : circuit $C_{1}: n \rightarrow n-1 \rightarrow \ldots \rightarrow 1 \rightarrow n$ of length $n$ and circuit $C_{2}: n-1 \rightarrow n-2 \rightarrow 1 \rightarrow n-1$ of length $n-1$. Note that only the longest circuit passes through vertex $n$. Furthermore, if $n>2$ then $\operatorname{gcd}(n-1, n)=1$. Any circuit that passes through vertex $n$ can be considered as a concatenation of $\alpha$ times $C_{1}$, a path from vertex $n$ to a vertex $t$ in $C_{2}, \beta$ times $C_{2}$, and a path from $t$ to $n$ for some nonnegative integers $\alpha$ and $\beta$. The length of this circuit is equal to $n+\alpha n+\beta(n-1)$. By Lemma 2.1 the smallest integer $N$ such that for any integer 


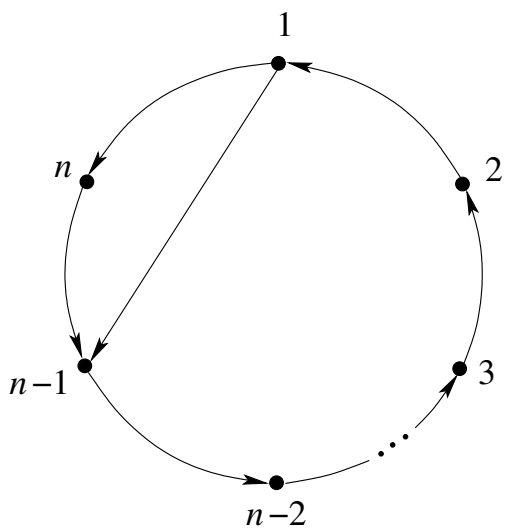

FIG. 3.2. The precedence graph of the matrix $\hat{A}$ of Example 3.4.

$p \geq N$ there exist nonnegative integers $\alpha$ and $\beta$ such that $p=\alpha n+\beta(n-1)$ is given by $N=(n-1)(n-2)$. This implies that $(n-1)(n-2)-1$ cannot be written as $\gamma n+\delta(n-1)$ with $\gamma, \delta \in \mathbb{N}$. This implies that there does not exist a circuit of length $n+(n-1)(n-2)-1=(n-1)^{2}$ that passes through vertex $n$. Hence, $\left(A^{\otimes^{(n-1)^{2}}}\right)_{n n}=\mathcal{O}$ and thus $A^{\otimes^{(n-1)^{2}}} \neq \mathcal{E} . \quad \diamond$

Let $A \in \mathbb{B}^{n \times n}$. If $\hat{A}=P \otimes A \otimes P^{T}$ is the Frobenius normal form of $A$, then we have $A=P^{T} \otimes \hat{A} \otimes P$. Hence,

$$
A^{\otimes^{k}}=\left(P^{T} \otimes \hat{A} \otimes P\right)^{\otimes^{k}}=P^{T} \otimes \hat{A}^{\otimes^{k}} \otimes P
$$

for all $k \in \mathbb{N}$. Therefore, we may consider without loss of generality the sequence $\left\{\hat{A}^{\otimes^{k}}\right\}_{k=1}^{\infty}$ instead of the sequence $\left\{A^{\otimes^{k}}\right\}_{k=1}^{\infty}$. Furthermore, since the transformation from $A$ to $\hat{A}$ corresponds to a simultaneous reordering of the rows and columns of $A$ (or to a reordering of the vertices of $\mathcal{G}(A)$ ), we have $c(A)=c(\hat{A})$.

THEOREM 3.5. Let $\hat{A} \in \mathbb{B}^{n \times n}$ be a matrix of the form (1) where the matrices $\hat{A}_{11}, \hat{A}_{22}, \ldots, \hat{A}_{l l}$ are irreducible and such that $c(\hat{A})=1$. Define sets $\alpha_{1}, \alpha_{2}, \ldots, \alpha_{l}$ such that $\hat{A}_{\alpha_{i} \alpha_{j}}=\hat{A}_{i j}$ for all $i, j$ with $i \leq j$. Let $n_{i}=\# \alpha_{i}$ for all $i$. Define:

$$
\lambda_{i}= \begin{cases}\mathbf{0} & \text { if } \hat{A}_{i i}=[\mathbf{0}] \\ \mathbf{1} & \text { otherwise }\end{cases}
$$

for $i=1,2, \ldots, l$. Define

$$
\begin{aligned}
& S_{i j}=\left\{\left\{i_{0}, i_{1}, \ldots, i_{s}\right\} \subseteq\{1,2, \ldots, l\} \mid i=i_{0}<i_{1}<\ldots<i_{s}=j\right. \text { and } \\
& \hat{A}_{i_{r} i_{r+1}}\neq \mathcal{O} \text { for } r=0,1, \ldots, s-1\}
\end{aligned}
$$

for all $i, j$ with $i<j$.

Let $\lambda_{i i}=\lambda_{i}$ and $k_{i i}=\left(n_{i}-1\right)^{2}+1$ for $i=1,2, \ldots, n$. Define

$$
\begin{aligned}
\Gamma_{i j} & =\left\{t \mid \exists \gamma \in S_{i j} \text { such that } t \in \gamma\right\} \\
\lambda_{i j} & = \begin{cases}\bigoplus_{t \in \Gamma_{i j}} \lambda_{t} & \text { if } \Gamma_{i j} \neq \emptyset \\
\mathcal{O} & \text { otherwise }\end{cases}
\end{aligned}
$$




$$
\begin{aligned}
t_{i j} & = \begin{cases}\arg \min \left\{n_{t} \mid t \in \Gamma_{i j} \text { and } \lambda_{t t} \neq \mathcal{O}\right\} & \text { if } \lambda_{i j} \neq \mathcal{0} \\
0 & \text { otherwise }\end{cases} \\
k_{i j} & = \begin{cases}\sum_{t \in \Gamma_{i j}} n_{t}+k_{t_{i j} t_{i j}} & \text { if } \lambda_{i j} \neq \mathbf{0} \\
t \neq t_{i j} & \text { if } \Gamma_{i j} \neq \emptyset \text { and } \lambda_{i j}=\mathcal{O} \\
\# \Gamma_{i j} & \text { if } \Gamma_{i j}=\emptyset \\
1 & \text { i }\end{cases}
\end{aligned}
$$

for all $i, j$ with $i<j$. Then we have for all $i, j$ with $i \leq j$ :

$$
\left(\hat{A}^{\otimes^{k}}\right)_{\alpha_{i} \alpha_{j}}=\left\{\begin{array}{ll}
\mathcal{E}_{n_{i} \times n_{j}} & \text { if } \lambda_{i j} \neq \mathcal{0} \\
\mathcal{O}_{n_{i} \times n_{j}} & \text { if } \lambda_{i j}=\mathcal{0}
\end{array} \quad \text { for all } k \geq k_{i j} .\right.
$$

For all $i, j$ with $i>j$ we have

$$
\left(\hat{A}^{\otimes^{k}}\right)_{\alpha_{i} \alpha_{j}}=\mathcal{O}_{n_{i} \times n_{j}} \quad \text { for all } k \in \mathbb{N} .
$$

REMARK 3.6. Note that $\hat{A}_{i j}$ is an $n_{i}$ by $n_{j}$ matrix for all $i, j$.

Let us now give a graphical interpretation of the sets $S_{i j}$ and $\Gamma_{i j}$.

Let $C_{i}$ be the m.s.c.s. of $\mathcal{G}(\hat{A})$ that corresponds to $\hat{A}_{i i}$ for $i=1,2 \ldots, l$. So $\alpha_{i}$ is the set of vertices of $C_{i}$.

If $\left\{i_{0}=i, i_{1}, \ldots, i_{s-1}, i_{s}=j\right\} \in S_{i j}$ then there exists a path from a vertex in $C_{i_{r}}$ to a vertex in $C_{i_{r-1}}$ for each $r=1,2, \ldots, s$. Since each m.s.c.s. $C_{i}$ of $\mathcal{G}(\hat{A})$ either is strongly connected or consists of only one vertex, this implies that there exists a path from a vertex in $C_{j}$ to a vertex in $C_{i}$ that passes through $C_{i_{s-1}}, C_{i_{s-2}}, \ldots, C_{i_{1}}$. If $S_{i j}=\emptyset$ then there does not exist any path from a vertex in $C_{j}$ to a vertex in $C_{i}$. The set $\Gamma_{i j}$ is the set of indices of the m.s.c.s.'s of $\mathcal{G}(\hat{A})$ through which some path from a vertex of $C_{j}$ to a vertex of $C_{i}$ passes.

If $S_{i j} \neq \emptyset$ then $C_{t_{i j}}$ is the smallest m.s.c.s. of $\mathcal{G}(A)$ that contains a circuit and through which some path from a vertex of $C_{j}$ to a vertex of $C_{i}$ passes $^{3}$.

Proof. Proof of Theorem 3.5.

Let $C_{i}$ be the m.s.c.s. of $\mathcal{G}(\hat{A})$ that corresponds to $\hat{A}_{i i}$ for $i=1,2 \ldots, l$. Since $\hat{A}_{\alpha_{i} \alpha_{j}}=$ $\mathcal{O}$ if $i>j$, there are no $\operatorname{arcs}$ from any vertex of $C_{j}$ to a vertex in $C_{i}$. As a consequence, (5) holds if $i>j$.

Note that $c\left(\hat{A}_{i i}\right)=1$ for all $i \in\{1,2, \ldots, l\}$ with $\hat{A}_{i i} \neq[\mathbf{o}]$ since $c(\hat{A})=1$ and since each $\hat{A}_{i i}$ corresponds to an m.s.c.s. of $\mathcal{G}(\hat{A})$.

If $l=1$ then $\hat{A}$ is irreducible and then (4) holds by Theorem 3.3. It is easy to verify that (4) holds if $i=j$.

From now on we assume that $l>1$ and $i<j$.

If $\Gamma_{i j}=\emptyset$ then there does not exist a path from a vertex in $C_{j}$ to a vertex in $C_{i}$. Hence, $\left(\hat{A}^{\otimes^{k}}\right)_{\alpha_{i} \alpha_{j}}=\mathcal{O}$ for all $k \in \mathbb{N}$.

If $\Gamma_{i j} \neq \emptyset$ and $\lambda_{i j}=\mathcal{O}$ then we have $\hat{A}_{t t}=[\mathcal{O}]$ for all $t \in \Gamma_{i j}$. So there exist paths from a vertex in $C_{j}$ to a vertex in $C_{i}$, but each path passes only through m.s.c.s.'s that consist of one vertex and contain no loop. Such a path passes through at most

\footnotetext{
${ }^{3}$ Or more precisely: if $S_{i j} \neq \emptyset$ then $C_{t_{i j}}$ belongs to the set of the smallest m.s.c.s.'s of $\mathcal{G}(A)$ that contain a circuit and through which some path from a vertex of $C_{j}$ to a vertex of $C_{i}$ passes.
} 


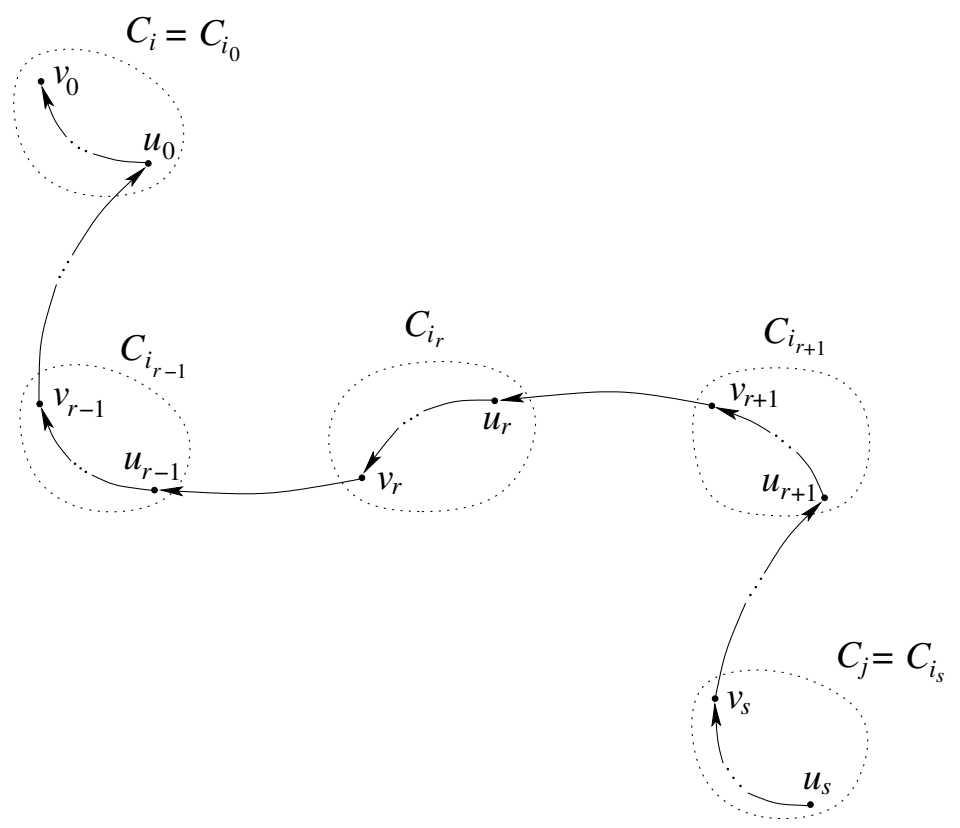

FIG. 3.3. Illustration of the proof of Theorem 3.5. There exists a path from vertex $u_{s}$ of m.s.c.s. $C_{j}$ to vertex $v_{0}$ of m.s.c.s. $C_{i}$ that passes through the m.s.c.s.'s $C_{i_{s-1}}, C_{i_{s-2}}, \ldots, C_{i_{1}}$.

$\# \Gamma_{i j}$ of such m.s.c.s.'s $\left(C_{j}\right.$ and $C_{i}$ included). This implies that there does not exist a path with a length that is larger than or equal to $\# \Gamma_{i j}$ from a vertex in $C_{j}$ to a vertex in $C_{i}$. Hence, we have $\left(A^{\otimes^{k}}\right)_{\alpha_{i} \alpha_{j}}=\mathcal{O}$ for all $k \geq \# \Gamma_{i j}$.

From now on we assume that $l>1, i<j, \Gamma_{i j} \neq \emptyset$ and $\lambda_{i j} \neq 0$. Then there exists a set $\left\{i_{0}, i_{1}, \ldots, i_{s}\right\} \subseteq\{1,2, \ldots, l\}$ with $t_{i j} \in\left\{i_{0}, i_{1}, \ldots, i_{s}\right\}$ and there exist indices $u_{r} \in \alpha_{i_{r}}, v_{r+1} \in \alpha_{i_{r+1}}$ for $r=0,1, \ldots, s-1$ such that $i=i_{0}<i_{1}<\ldots<i_{s}=j$ and $\hat{A}_{u_{r} v_{r+1}} \neq \mathrm{O}$ for each $r$. So there exists an arc from vertex $v_{r+1}$ of $C_{i_{r+1}}$ to vertex $u_{r}$ of $C_{i_{r}}$ for each $r \in\{0,1, \ldots, s-1\}$. Select an arbitrary vertex $u_{s}$ of $C_{i_{s}}=C_{j}$ and an arbitrary vertex $v_{0}$ of $C_{i_{0}}=C_{i}$. Note that $s \in\{1,2, \ldots, j-i\}$. Let $r \in\{0,1, \ldots, s\}$. Recall that the only Boolean zero matrix that is irreducible is the 1 by 1 Boolean zero matrix [0]. Now we distinguish between two cases:

- If $\hat{A}_{i_{r} i_{r}}=[\mathbf{o}]$ then we have $n_{i_{r}}=1$ and $u_{r}=v_{r}$. So in this case we could say that there exists an empty path of length $l_{r}=0$ from vertex $u_{r}$ to vertex $v_{r}$ of $C_{i_{r}}$.

- On the other hand, if $\hat{A}_{i_{r} i_{r}} \neq[0]$, then there exists a (possibly empty) path of length $l_{r} \leq n_{i_{r}}-1$ from vertex $u_{r}$ to vertex $v_{r}$ of $C_{i_{r}}$ since $\mathcal{G}\left(\hat{A}_{i_{r} i_{r}}\right)$ is strongly connected. If $u_{r}=v_{r}$ then this path is empty and has length 0 .

So for each $r \in\{0,1, \ldots, s\}$ there exists a (possible empty) path of length $l_{r} \leq n_{i_{r}}-1$ from vertex $u_{r}$ to vertex $v_{r}$ of $C_{i_{r}}$.

Let $\tilde{t}=i_{\tilde{r}}=t_{i j}$. Clearly, we have $\hat{A}_{i_{\tilde{r}} i_{\tilde{r}}} \neq[\mathbf{o}]$. Since $\hat{A}_{i_{\tilde{r}} i_{\tilde{r}}}$ is irreducible and since $c\left(\hat{A}_{i_{\tilde{r}} i_{\tilde{r}}}\right)=1$, it follows from Theorem 3.3 that there exists a path of length $k$ from 
vertex $u_{\tilde{r}}$ to vertex $v_{\tilde{r}}$ of $C_{i_{\tilde{r}}}$ for any $k \geq k_{i_{\tilde{r}} i_{\tilde{r}}}=k_{\tilde{t} \tilde{t}}$. Note that $\# \Gamma_{i j} \geq s+1$. Hence,

$$
k_{i j}=\sum_{\substack{t \in \Gamma_{i j} \\ t \neq \tilde{t}}} n_{t}+k_{\tilde{t} \tilde{t}}=\sum_{\substack{t \in \Gamma_{i j} \\ t \neq \tilde{t}}}\left(n_{t}-1\right)+\left(\# \Gamma_{i j}-1\right)+k_{\tilde{t} \tilde{t}} \geq \sum_{\substack{r=0 \\ r \neq \tilde{r}}}^{s} l_{r}+s+k_{\tilde{t} \tilde{t}}
$$

So if we have an integer $k \geq k_{i j}$ then we can decompose it as

$$
k=l_{0}+l_{1}+\ldots+l_{\tilde{r}-1}+l_{\tilde{r}+1}+\ldots+l_{s}+s+k_{\tilde{t} \tilde{t}}+\tilde{k}
$$

with $\tilde{k} \in \mathbb{N}$. By Theorem 3.3 there exists a path of length $k_{\tilde{t} \tilde{t}}+\tilde{k}$ from $u_{\tilde{r}}$ to $v_{\tilde{r}}$ in $C_{i_{\tilde{r}}}$ for each $\tilde{k} \in \mathbb{N}$. This implies that there exists a path from vertex $u_{s}$ to vertex $v_{0}$ of length $k$ in $\mathcal{G}(\hat{A})$. This path consists of the concatenation of paths of length $l_{r}$ from vertex $u_{r}$ to vertex $v_{r}$ of $C_{i_{r}}$ for $r=0,1, \ldots, \tilde{r}-1, \tilde{r}+1, \ldots, s$, paths of length 1 from vertex $v_{r+1}$ of $C_{i_{r+1}}$ to vertex $u_{r}$ of $C_{i_{r}}$ for $r=0,1, \ldots, s-1$ and a path of length $k_{\tilde{t} \tilde{t}}+\tilde{k}$ from vertex $u_{\tilde{r}}$ to vertex $v_{\tilde{r}}$ of $C_{i_{\tilde{r}}}$ (See Figure 3.3 ). This implies that $\left(\hat{A}^{\otimes}\right)_{v_{0} u_{s}}=1$ for all $k \geq k_{i j}$. Since $u_{s}$ is an arbitrary vertex of $C_{j}$ and since $v_{0}$ is an arbitrary vertex of $C_{i}$, this implies that $\left(\hat{A}^{\otimes^{k}}\right)_{\alpha_{i} \alpha_{j}}=\mathcal{E}$ for all $k \geq k_{i j}$.

So (4) also holds if $\lambda_{i j} \neq 0$.

ExAMPLE 3.7. Consider the following matrix:

$$
\hat{A}=\left[\begin{array}{lll|l}
0 & 1 & 0 & 0 \\
1 & 0 & 1 & 0 \\
1 & 0 & 0 & 1 \\
\hline 0 & 0 & 0 & 0
\end{array}\right]
$$

This matrix is in Frobenius normal form and its block structure is indicated by the vertical and horizontal lines. The precedence graph of $\hat{A}$ is represented in Figure 3.4. Using the notations and definitions of Theorem 3.5, we have $l=2$,

$$
\hat{A}_{11}=\left[\begin{array}{lll}
0 & 1 & 0 \\
1 & 0 & 1 \\
1 & 0 & 0
\end{array}\right], \hat{A}_{12}=\left[\begin{array}{l}
0 \\
0 \\
1
\end{array}\right] \text { and } \hat{A}_{22}=[0]
$$

Furthermore, $\alpha_{1}=\{1,2,3\}, \alpha_{2}=\{4\}, n_{1}=3, n_{2}=1, \lambda_{1}=\lambda_{11}=\mathbf{1}, \lambda_{2}=\lambda_{22}=\mathbf{o}$, $S_{12}=\{\{1,2\}\}, \Gamma_{12}=\{1,2\}, \lambda_{12}=\mathbf{1}, t_{12}=1, k_{11}=5, k_{22}=1$ and $k_{12}=1+5=6$. We have

$$
\begin{aligned}
& \hat{A}^{\otimes}=\left[\begin{array}{lll|l}
1 & 0 & 1 & 0 \\
1 & 1 & 0 & 1 \\
0 & 1 & 0 & 0 \\
\hline 0 & 0 & 0 & 0
\end{array}\right], \quad \hat{A}^{\otimes}=\left[\begin{array}{lll|l}
1 & 1 & 0 & 1 \\
1 & 1 & 1 & 0 \\
1 & 0 & 1 & 0 \\
\hline 0 & 0 & 0 & 0
\end{array}\right], \quad \hat{A}^{\otimes}=\left[\begin{array}{lll|l}
1 & 1 & 1 & 0 \\
1 & 1 & 1 & 1 \\
1 & 1 & 0 & 1 \\
\hline 0 & 0 & 0 & 0
\end{array}\right] \\
& \hat{A}^{\otimes^{5}}=\left[\begin{array}{lll|l}
1 & 1 & 1 & 1 \\
1 & 1 & 1 & 1 \\
1 & 1 & 1 & 0 \\
\hline 0 & 0 & 0 & 0
\end{array}\right], \quad \hat{A}^{\otimes^{6}}=\left[\begin{array}{lll|l}
1 & 1 & 1 & 1 \\
1 & 1 & 1 & 1 \\
1 & 1 & 1 & 1 \\
\hline 0 & 0 & 0 & 0
\end{array}\right]=A^{\otimes^{7}}=\ldots
\end{aligned}
$$

Note that $\hat{A}_{11}$ is a matrix of the form (3). So the smallest $K_{11}$ for which $\hat{A}_{11}^{\otimes^{k}}=\mathcal{E}$ for all $k \geq K_{11}$ is equal to $k_{11}=5$. Furthermore, the smallest $K_{12}$ such that $\left(\hat{A}^{\otimes^{k}}\right)_{\alpha_{1} \alpha_{2}}=\mathcal{E}$ 


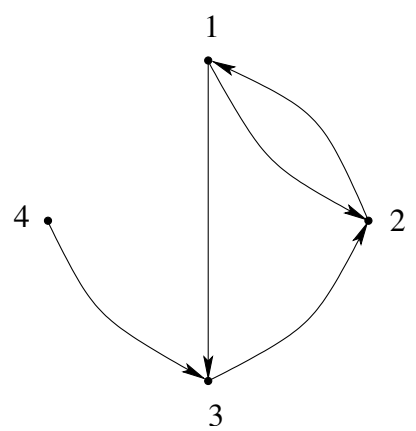

FIG. 3.4. The precedence graph of the matrix $\hat{A}$ of Example 3.7.

for all $k \geq K_{12}$ is equal to $k_{12}=6$. So for the matrix $\hat{A}$ of this example all the bounds $k_{i j}$ that appear in Theorem 3.5 are tight.

It is easy to verify that for a matrix of the form

$$
\left[\begin{array}{ccccc|c}
0 & 1 & 0 & \ldots & 0 & 0 \\
0 & 0 & 1 & \ldots & 0 & 0 \\
\vdots & \vdots & \vdots & \ddots & \vdots & \vdots \\
1 & 0 & 0 & \ldots & 1 & 0 \\
1 & 0 & 0 & \ldots & 0 & 1 \\
\hline 0 & 0 & 0 & \ldots & 0 & 0
\end{array}\right]
$$

all the bounds $k_{i j}$ that appear in Theorem 3.5 are tight.

Lemma 3.8. Let $A \in \mathbb{B}^{n \times n}$ with $c(A)=1$. Then we have $A^{\otimes^{k+1}}=A^{\otimes^{k}}$ for all $k \geq(n-1)^{2}+1$.

Proof. If $A$ is irreducible, then we have $A^{\otimes^{k+1}}=A^{\otimes^{k}}$ for all $k \geq(n-1)^{2}+1$ by Theorem 3.3.

So from now on we assume that $A$ is not irreducible. Let $\hat{A}=P \otimes A \otimes P^{T}$ be the Frobenius normal form of $A$. Assume that $\hat{A}$ is of the form (1) where the $\hat{A}_{i i}$ 's are square and irreducible. Let the numbers $\lambda_{i j}, n_{i}, t_{i j}, k_{i j}$ and the sets $\alpha_{i}$ and $\Gamma_{i j}$ be defined as in Theorem 3.5.

We have $k_{i i}=\left(n_{i}-1\right)^{2}+1 \leq(n-1)^{2}+1$ for all $i$.

Let us now prove that $k_{i j} \leq(n-1)^{2}+1$ for all $i, j$ with $i<j$. Consider indices $i, j \in\{1,2, \ldots, l\}$ with $i<j$.

- If $\Gamma_{i j}=\emptyset$ then we have $k_{i j}=1 \leq(n-1)^{2}+1$ for all $n \in \mathbb{N}_{0}$. have

- We have $n \leq(n-1)^{2}+1$ for all $n \in \mathbb{N}_{0}$. So if $\Gamma_{i j} \neq \emptyset$ and $\lambda_{i j}=\mathcal{O}$ then we

$$
k_{i j}=\# \Gamma_{i j} \leq j-i+1 \leq n \leq(n-1)^{2}+1
$$

- Since $n_{t} \geq 1$ for each $t \in\{1,2, \ldots, l\}$ and since $l \geq 1$, we have

$$
\sum_{\substack{t, s=1 \\ t \neq s}}^{l} n_{t} n_{s} \geq \sum_{\substack{t, s=1 \\ t \neq s}}^{l} 1 \geq l^{2}-l \geq l-1 .
$$


Hence,

$$
l \leq \sum_{\substack{t, s=1 \\ t \neq s}}^{l} n_{t} n_{s}+1
$$

So if $\lambda_{i j} \neq \mathrm{O}$ then we have

$$
\begin{aligned}
& k_{i j}=\sum_{\substack{t \in \Gamma_{i j} \\
t \neq t_{i j}}} n_{t}+k_{t_{i j} t_{i j}} \\
& \leq \sum_{t=1}^{l} k_{t t} \quad\left(\text { since } k_{i i}=\left(n_{i}-1\right)^{2}+1 \geq n_{i} \text { for each } i\right) \\
& \leq \sum_{t=1}^{l}\left(\left(n_{t}-1\right)^{2}+1\right) \\
& \leq \sum_{t=1}^{l}\left(n_{t}^{2}-2 n_{t}+2\right) \\
& \leq \sum_{t=1}^{l} n_{t}^{2}-2 \sum_{t=1}^{l} n_{t}+2 l \\
& \leq \sum_{t=1}^{l} n_{t}^{2}-2 n+2 l \\
& \leq \sum_{t=1}^{l} n_{t}^{2}-2 n+2\left(\sum_{\substack{t, s=1 \\
t \neq s}}^{l} n_{t} n_{s}+1\right) \quad(\text { by }(6)) \\
& \leq\left(\sum_{t=1}^{l} n_{t}^{2}+2 \sum_{\substack{t, s=1 \\
t \neq s}}^{l} n_{t} n_{s}\right)-2 n+2 \\
& \leq\left(\sum_{t=1}^{l} n_{t}\right)^{2}-2 n+1+1 \\
& \leq n^{2}-2 n+1+1 \\
& \leq(n-1)^{2}+1 \text {. }
\end{aligned}
$$

Hence, $k_{i j} \leq(n-1)^{2}+1$ for all $i, j$ with $i \leq j$. As a consequence, it follows from Theorem 3.5 that $\left(\hat{A}^{\otimes^{k+1}}\right)_{\alpha_{i} \alpha_{j}}=\left(\hat{A}^{\otimes^{k}}\right)_{\alpha_{i} \alpha_{j}}$ for all $k \geq(n-1)^{2}+1$ and for all $i, j \in$ $\{1,2, \ldots, l\}$. Hence, $\hat{A}^{\otimes^{k+1}}=\hat{A}^{\otimes^{k}}$ for all $k \geq(n-1)^{2}+1$. Since $A^{\otimes^{k}}=P^{T} \otimes \hat{A}^{\otimes^{k}} \otimes P$, this implies that $A^{\otimes^{k+1}}=A^{\otimes^{k}}$ for all $k \geq(n-1)^{2}+1$.

3.3. Boolean matrices with a cyclicity that is larger than or equal to 1. Lemma 3.9. Let $A \in \mathbb{B}^{n \times n}$ be an irreducible matrix with $c(A) \geq 2$ and let $i, j \in\{1,2, \ldots, n\}$. Then there exists a (possibly empty) path $P_{i j}$ from $j$ to $i$ in $\mathcal{G}(A)$ 
that passes through at least one vertex of each (elementary) circuit of $\mathcal{G}(A)$ and that has a length that is less than or equal to $\frac{n^{2}-1}{2}$.

Proof. Since the cyclicity of $A$ is larger than or equal to 2 , there are no loops in $\mathcal{G}(A)$. Hence, $A$ contains at least one circuit. Since $A$ is irreducible, this implies that $j$ has to belong to an elementary circuit of $\mathcal{G}(A)$. Since the length of any elementary circuit of $\mathcal{G}(A)$ is larger than or equal to 2 , there exists a set $S=\left\{i_{1}=j, i_{2}, \ldots, i_{m}\right\} \subseteq$ $\{1,2, \ldots, n\}$ with $m \leq\left\lceil\frac{n}{2}\right\rceil$ such that any (elementary) circuit of $\mathcal{G}(A)$ contains at least one vertex that belongs to $S$. Define $i_{m+1}=i$. Since $\mathcal{G}(A)$ is strongly connected there exists a (possibly empty) path $P_{k}$ with length $l_{k} \leq n-1$ from vertex $i_{k}$ to vertex $i_{k+1}$ for each $k \in\{1,2, \ldots, m\}$. Let $l_{k}$ be the length of $P_{k}$. There exists a path $P_{i j}$ from $j$ to $i$ that contains at least one vertex of each (elementary) circuit of $\mathcal{G}(A)$ : this path consists of the concatenation of $P_{1}, P_{2}, \ldots, P_{m}$. If $l_{i j}$ is the length of $P_{i j}$, then we have

$$
l_{i j}=l_{1}+\ldots+l_{m} \leq m(n-1) \leq\left(\frac{n+1}{2}\right)(n-1) \leq \frac{n^{2}-1}{2} .
$$

REMARK 3.10. Note that we could have derived an upper bound that is more tight in Lemma 3.9. The upper bound of Lemma 3.9 will be used in the proof of Theorem 3.11. However, in that proof we shall also use Lemmas 2.2 and 2.3 which also yield upper bounds, and therefore we do not refine the upper bound of Lemma 3.9. $\diamond$

Theorem 3.11. Let $A \in \mathbb{B}^{n \times n}$ be irreducible and let $c=c(A)>0$. If we define

$$
k_{n, c}= \begin{cases}(n-1)^{2}+1 & \text { if } c=1 \\ \max \left(n-1, \frac{n^{2}-1}{2}+\frac{n^{2}}{c}-3 n+2 c\right) & \text { if } c>1,\end{cases}
$$

then we have

(8) $A^{\otimes^{k+c}}=A^{\otimes^{k}}$ and $A^{\otimes^{k}} \oplus A^{\otimes^{k+1}} \oplus \ldots \oplus A^{\otimes^{k+c-1}}=\mathcal{E}_{n \times n} \quad$ for all $k \geq k_{n, c}$.

Proof. From Theorem 3.3 it follows that (8) holds if $c$ is equal to 1 . Furthermore, if the first part of (8) holds, then the second part also holds since $A$ is irreducible.

From now on we assume that $c>1$. Let $i, j \in\{1,2, \ldots, n\}$.

Let $C_{1}, C_{2}, \ldots, C_{m}$ be the elementary circuits of $\mathcal{G}(A)$. Let $l_{i}$ be the length of $C_{i}$ for $i=1,2, \ldots, m$. Since $A$ is irreducible, we have $c=\operatorname{gcd}\left(l_{1}, l_{2}, \ldots, l_{m}\right)$. Hence, there exist positive integers $w_{1}, w_{2}, \ldots, w_{m}$ such that $w_{i} c=l_{i}$ for each $i$ and such that $\operatorname{gcd}\left(w_{1}, w_{2}, \ldots, w_{m}\right)=1$.

First we consider the case where there is only one elementary circuit or where all the elementary circuits have the same length. Hence, $c=l_{1}$. Since $A$ is irreducible, both $i$ and $j$ have to belong to some elementary circuit. We may assume without loss of generality that $j$ belongs to $C_{1}$. Since $A$ is irreducible there exist paths from vertex $j$ to vertex $i$ of $\mathcal{G}(A)$. Let $P_{i j}$ be the shortest (possibly empty) path from $j$ to $i$ and let $l_{i j}$ be the length of this path. We have $l_{i j} \leq n-1$ (Note that $l_{i j}=0$ if $i$ is equal to $j$.). For any integer $k \in \mathbb{N}$ there exists a path of length $l_{i j}+k c$ from $j$ to $i$ : this path consists of $k$ times $C_{1}$ followed by $P_{i j}$. Hence $\left(A^{\otimes^{l_{i j}+k c}}\right)_{i j}=\mathbf{1}$ for all $k \geq 0$. Let $l \in \mathbb{N}$ with $l \geq n-1$. Now there are two possibilities. If $l$ can be written as $l=l_{i j}+k c$ for some $k \in \mathbb{N}$, then we have $\left(A^{\otimes^{l}}\right)_{i j}=1$. If $l$ cannot be written as $l=l_{i j}+k c$ for any $k \in \mathbb{N}$, then it follows from Lemma 2.7 that there does not exist a path from $j$ to 
$i$ and then we have $\left(A^{\otimes^{l}}\right)_{i j}=\mathbf{0}$.

This implies that (8) holds if all the elementary circuits of $\mathcal{G}(A)$ have the same length.

From now on we assume that there exist at least two elementary circuits in $\mathcal{G}(A)$ that have different lengths.

Since $A$ is irreducible it follows from Lemma 3.9 that there exists a (possibly empty) path $P_{i j}$ from vertex $j$ to vertex $i$ of $\mathcal{G}(A)$ with length $l_{i j} \leq \frac{n^{2}-1}{2}$ that passes through at least one vertex of each elementary circuit of $\mathcal{G}(A)$. For each circuit $C_{k}$ we select one vertex $v_{k}$ that belongs to the path $P_{i j}$. Let $l$ be an integer that can be written as $l=l_{i j}+p c$ with $p \geq g\left(w_{1}, w_{2}, \ldots, w_{m}\right)+1$. Since $\operatorname{gcd}\left(w_{1}, w_{2}, \ldots, w_{m}\right)=1$, there exist nonnegative integers $\alpha_{1}, \alpha_{2}, \ldots, \alpha_{m}$ such that $p=\alpha_{1} w_{1}+\alpha_{2} w_{2}+\ldots+\alpha_{m} w_{m}$. As a consequence, we have

$$
l=l_{i j}+\alpha_{1} w_{1} c+\alpha_{2} w_{2} c+\ldots+\alpha_{m} w_{m} c=l_{i j}+\alpha_{1} l_{1}+\alpha_{2} l_{2}+\ldots+\alpha_{m} l_{m} .
$$

So there exists a path of length $l$ from $j$ to $i$ : this path consists of the concatenation of $P_{i j}$ and $\alpha_{k}$ times the circuit $C_{k}\left(v_{k} \rightarrow \ldots \rightarrow v_{k}\right)$ for $k=1,2, \ldots, m$. Hence, $\left(A^{\otimes^{l_{i j}+p c}}\right)_{i j}=\mathbf{1}$ for all $p \geq g\left(w_{1}, w_{2}, \ldots, w_{m}\right)+1$.

Let us now determine an upper bound for $g\left(w_{1}, w_{2}, \ldots, w_{m}\right)$. If $w_{s}=w_{t}$ for some $s, t \in$ $\{1,2, \ldots, m\}$ with $s \neq t$, then $g\left(w_{1}, w_{2}, \ldots, w_{m}\right)=g\left(w_{1}, w_{2}, \ldots, w_{s-1}, w_{s+1}, \ldots, w_{m}\right)$. Therefore, we may assume without loss of generality that all the $w_{i}$ 's are different and thus also that $w_{1}<w_{2}<\ldots<w_{m}$.

Since there are at least two elementary circuits in $\mathcal{G}(A)$ that have different lengths, we have $m \geq 2$. We have $w_{k}=\frac{l_{k}}{c} \leq \frac{n}{c}$ for all $k$. Hence, $w_{m} \leq \frac{n}{c}$ and $w_{1} \leq \frac{n}{c}-(m-1) \leq$ $\frac{n}{c}-1$ since $w_{1}<w_{2} \ldots<w_{m}$ and $m \geq 2$. As a consequence, we have

$$
\begin{aligned}
g\left(w_{1}, w_{2}, \ldots, w_{m}\right) & \leq\left(w_{1}-1\right)\left(w_{m}-1\right)-1 \quad \text { (by Lemma 2.2) } \\
& \leq\left(\frac{n}{c}-2\right)\left(\frac{n}{c}-1\right)-1 \\
& \leq\left(\frac{n}{c}\right)^{2}-3 \frac{n}{c}+1 .
\end{aligned}
$$

If we define

$$
K=\frac{n^{2}-1}{2}+\left(\left(\frac{n}{c}\right)^{2}-3 \frac{n}{c}+2\right) c
$$

then we have $l_{i j}+\left(g\left(w_{1}, w_{2}, \ldots, w_{m}\right)+1\right) c \leq K$. So if we have an integer $l$ that is larger than $K$ then it can either be written as $l=l_{i j}+p c$ with $p \geq g\left(w_{1}, w_{2}, \ldots, w_{m}\right)+1$ and then $\left(A^{\otimes^{l}}\right)_{i j}=\mathbf{1}$, or $l$ cannot be written as $l=l_{i j}+p c$ for any $p \in \mathbb{N}$ and then it follows from Lemma 2.7 that there does not exist a path of length $l$ from $j$ to $i$, i.e., $\left(A^{\otimes^{l}}\right)_{i j}=\mathcal{O}$. Note that $K \leq k_{n, c}$.

Hence, (8) also holds in this case.

REMARK 3.12. In the proof of Theorem 3.11 we could also have used Lemma 2.3 to determine an upper bound for $g\left(w_{1}, w_{2}, \ldots, w_{m}\right)$. We have $m \geq 2$ and thus also $w_{m} \geq 2$. Furthermore, $w_{m-1} \leq \frac{n}{c}-1$. Hence,

$$
\begin{aligned}
g\left(w_{1}, w_{2}, \ldots, w_{m}\right) & \leq 2 w_{m-1}\left\lfloor\frac{w_{m}}{m}\right\rfloor-w_{m} \\
& \leq 2\left(\frac{n}{c}-1\right) \frac{n}{2 c}-2
\end{aligned}
$$




$$
\leq\left(\frac{n}{c}\right)^{2}-\frac{n}{c}-2
$$

In the second part of the proof of Theorem 3.11 we have $c \geq 2$. Since $A$ is irreducible, it follows from Lemma 2.6 that $c \leq n$. Hence, $1 \leq \frac{n}{c} \leq \frac{n}{2}$. It is easy to verify that the upper bound of (10) is less than the upper bound of (9) if $\frac{n}{c}<\frac{3}{2}$. However, if $\frac{n}{c}<\frac{3}{2}$, then we would have $w_{m-1} \leq \frac{1}{2}$, which is not possible. This implies that for combinations of $n$ and $c$ for which there are at least two elementary circuits in $\mathcal{G}(A)$ with different lengths, the upper bound of (9) is less than or equal to the upper bound of (10). $\diamond$

The Boolean sum of sequences is defined as follows. Consider sequences $g_{i}=\left\{\left(g_{i}\right)_{k}\right\}_{k=1}^{\infty}$ for $i=1,2, \ldots, m$ with $\left(g_{i}\right)_{k} \in \mathbb{B}$ for all $i, k$. The sequence $g=g_{1} \oplus g_{2} \oplus \ldots \oplus g_{m}$ is defined by $g_{k}=\left(g_{1}\right)_{k} \oplus\left(g_{2}\right)_{k} \oplus \ldots \oplus\left(g_{m}\right)_{k}$ for all $k \in \mathbb{N}_{0}$.

Lemma 3.13. Consider sequences $g_{i}=\left\{\left(g_{i}\right)_{k}\right\}_{k=1}^{\infty}$ for $i=1,2, \ldots, m$ with $\left(g_{i}\right)_{k} \in$ $\mathbb{B}$ for all $i, k$. Suppose that for each $i \in\{1,2, \ldots, m\}$ there exist integers $K_{i}, c_{i} \in \mathbb{N}_{0}$ such that

$$
\left(g_{i}\right)_{k+c_{i}}=\left(g_{i}\right)_{k} \quad \text { for all } k \geq K_{i} .
$$

If $K=\max _{i} K_{i}$ and

$$
c= \begin{cases}1 & \text { if } c_{i}=1 \text { and }\left(g_{i}\right)_{K_{i}}=\mathbf{1} \text { for some } i \in\{1,2, \ldots, m\} \\ \operatorname{lcm}\left(c_{1}, c_{2}, \ldots, c_{m}\right) & \text { otherwise },\end{cases}
$$

then the sequence $g=g_{1} \oplus g_{2} \oplus \ldots \oplus g_{m}$ satisfies $g_{k+c}=g_{k}$ for all $k \geq K$.

Proof. Note that (11) implies that

$$
\left(g_{i}\right)_{k+p c_{i}}=\left(g_{i}\right)_{k} \quad \text { for all } k \geq K \geq K_{i} \text { and for all } p \in \mathbb{N} .
$$

First we assume that there exists an index $i \in\{1,2, \ldots, m\}$ such that $c_{i}=1$ and $\left(g_{i}\right)_{K_{i}}=\mathbf{1}$. Then we have $\left(g_{i}\right)_{k}=\mathbf{1}$ for all $k \geq K_{i}$ and thus also $g_{k}=\mathbf{1}$ for all $k \geq K \geq K_{i}$.

From now on we assume that there does not exist any index $i \in\{1,2, \ldots, m\}$ such that $c_{i}=1$ and $\left(g_{i}\right)_{K_{i}}=\mathbf{1}$.

Since $c=\operatorname{lcm}\left(c_{1}, c_{2}, \ldots, c_{m}\right)$ there exist positive integers $w_{1}, w_{2}, \ldots, w_{m}$ such that $c=w_{i} c_{i}$ for $i=1,2, \ldots, m$. Consider an integer $k \geq K$. We have

$$
\begin{aligned}
g_{k+c} & =\bigoplus_{i=1}^{m}\left(g_{i}\right)_{k+c} \\
& =\bigoplus_{i=1}^{m}\left(g_{i}\right)_{k+w_{i} c_{i}} \\
& =\bigoplus_{i=1}^{m}\left(g_{i}\right)_{k} \\
& =g_{k} .
\end{aligned}
$$

EXAmPle 3.14. Consider the sequences

$$
\begin{aligned}
& g_{1}=\mathbf{1}, \mathcal{O}, \mathbf{1}, \mathcal{O}, \mathbf{1}, \mathcal{O}, \mathbf{1}, \mathcal{O}, \mathbf{1}, \mathcal{O}, \mathbf{1}, \mathcal{O}, \mathbf{1}, \mathcal{O}, \mathbf{1}, \mathcal{O}, \ldots \\
& g_{2}=\mathbf{1}, \mathfrak{O}, \mathcal{O}, \mathbf{1}, \mathfrak{O}, \mathcal{O}, \mathbf{1}, \mathfrak{O}, \mathcal{O}, \mathbf{1}, \mathfrak{O}, \mathcal{O}, \mathbf{1}, \mathfrak{O}, \mathcal{O}, \mathbf{1}, \ldots
\end{aligned}
$$


If we use the notation of Lemma 3.13 then we have $c_{1}=2, c_{2}=3$ and $K_{1}=K_{2}=1$. Hence, $c=\operatorname{lcm}(2,3)=6$ and $K=\max \left(K_{1}, K_{2}\right)=1$. We have

$$
g=g_{1} \oplus g_{2}=\mathbf{1}, \mathfrak{O}, \mathbf{1}, \mathbf{1}, \mathbf{1}, \mathfrak{O}, \mathbf{1}, \mathfrak{O}, \mathbf{1}, \mathbf{1}, \mathbf{1}, \mathfrak{O}, \mathbf{1}, \mathfrak{O}, \mathbf{1}, \mathbf{1}, \ldots
$$

It is easy to verify that $g_{k+6}=g_{k}$ for all $k \geq 1$. $\diamond$

THEOREM 3.15. Let $\hat{A} \in \mathbb{B}^{n \times n}$ be a matrix of the form (1) where the matrices $\hat{A}_{11}, \hat{A}_{22}, \ldots, \hat{A}_{l l}$ are irreducible. Define sets $\alpha_{1}, \alpha_{2}, \ldots, \alpha_{l}$ such that $\hat{A}_{\alpha_{i} \alpha_{j}}=\hat{A}_{i j}$ for all $i, j$ with $i \leq j$. Let $n_{i}=\# \alpha_{i}$ and $c_{i i}=c_{i}=c\left(\hat{A}_{i i}\right)$ for all $i$. Define:

$$
\lambda_{i}= \begin{cases}\mathbf{0} & \text { if } \hat{A}_{i i}=[\mathbf{0}] \\ \mathbf{1} & \text { otherwise }\end{cases}
$$

for $i=1,2, \ldots, l$. Define

$$
\begin{aligned}
& S_{i j}=\left\{\left\{i_{0}, i_{1}, \ldots, i_{s}\right\} \subseteq\{1,2, \ldots, l\} \mid\right. i=i_{0}<i_{1}<\ldots<i_{s}=j \text { and } \\
&\left.\hat{A}_{i_{r} i_{r+1}} \neq \mathcal{O} \text { for } r=0,1, \ldots, s-1\right\}
\end{aligned}
$$

for all $i, j$ with $i<j$.

Let $\lambda_{i i}=\lambda_{i}$ and $k_{i i}=k_{i}=k_{n_{i}, c_{i}}$ for $i=1,2, \ldots, n$ where $k_{n_{i}, c_{i}}$ is defined as in (7) with $k_{n_{i}, 0}=0$ by definition.

For each $i, j$ with $i<j$ we define for each $\gamma \in S_{i j}$ :

$$
\begin{aligned}
\delta_{\gamma} & =\left\{t \in \gamma \mid \lambda_{t} \neq 0\right\} \\
c_{\gamma} & = \begin{cases}\operatorname{gcd}\left\{c_{t} \mid t \in \delta_{\gamma}\right\} & \text { if } \delta_{\gamma} \neq \emptyset \\
1 & \text { otherwise }\end{cases}
\end{aligned}
$$

Define

$$
\begin{aligned}
& \Gamma_{i j}=\left\{t \mid \exists \gamma \in S_{i j} \text { such that } t \in \gamma\right\} \\
& \Delta_{i j}=\left\{t \mid t \in \Gamma_{i j} \text { and } \lambda_{t} \neq 0\right\} \\
& \lambda_{i j}= \begin{cases}\mathbf{1} & \text { if } \Delta_{i j} \neq \emptyset \\
\mathbf{0} & \text { otherwise }\end{cases} \\
& c_{i j}= \begin{cases}\operatorname{lcm}\left\{c_{\gamma} \mid \gamma \in S_{i j}\right\} & \text { if } \lambda_{i j} \neq \mathcal{0} \text { and } c_{\gamma} \neq 1 \text { for each } \gamma \in S_{i j} \text { with } \delta_{\gamma} \neq \emptyset \\
1 & \text { otherwise }\end{cases} \\
& r_{i j}= \begin{cases}\arg \max \left\{n_{t} \mid t \in \Delta_{i j}\right\} & \text { if } \lambda_{i j} \neq \mathrm{o} \\
1 & \text { otherwise }\end{cases}
\end{aligned}
$$

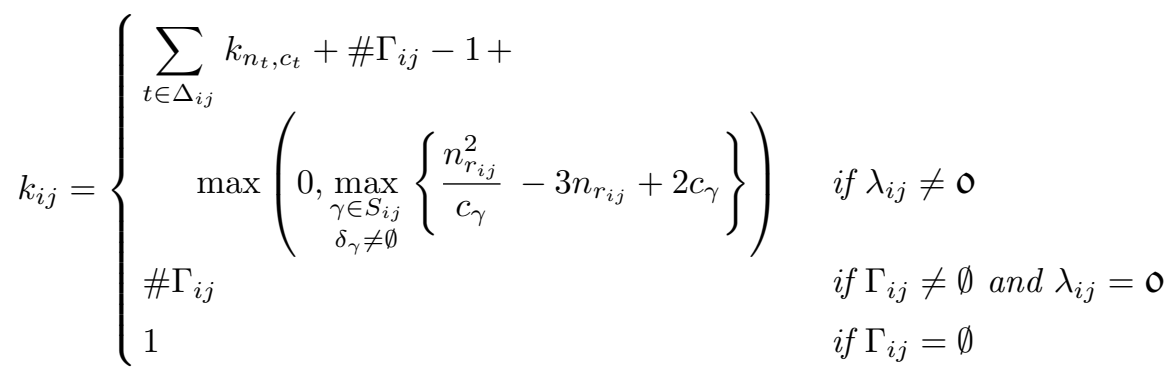


for all $i, j$ with $i<j$.

Then we have for all $i, j$ with $i \leq j$ :

$$
\left(\hat{A}^{\otimes^{k+c_{i j}}}\right)_{\alpha_{i} \alpha_{j}}=\left(\hat{A}^{\otimes^{k}}\right)_{\alpha_{i} \alpha_{j}} \quad \text { for all } k \geq k_{i j}
$$

and

$$
\left(\hat{A}^{{ }^{k}} \oplus \hat{A}^{\otimes^{k+1}} \oplus \ldots \oplus \hat{A}^{{ }^{k+c_{i j}-1}}\right)_{\alpha_{i} \alpha_{j}}= \begin{cases}\mathcal{E}_{n_{i} \times n_{j}} & \text { if } \lambda_{i j} \neq \mathcal{0} \\ \mathcal{O}_{n_{i} \times n_{j}} & \text { if } \lambda_{i j}=\mathcal{0}\end{cases}
$$

for all $k \geq k_{i j}$.

For all $i, j$ with $i>j$ we have

$$
\left(\hat{A}^{\otimes^{k}}\right)_{\alpha_{i} \alpha_{j}}=\mathcal{O}_{n_{i} \times n_{j}} \quad \text { for all } k \in \mathbb{N} .
$$

Proof. Let $C_{i}$ be the m.s.c.s. of $\mathcal{G}(\hat{A})$ that corresponds to $\hat{A}_{i i}$ for $i=1,2, \ldots, l$. Let $i, j \in\{1,2, \ldots, l\}$. In the proof of Theorem 3.15 is has already been proved that (15) holds if $i>j$, and that (13) and (14) hold if $i \leq j$ and $\Gamma_{i j}=\emptyset$, or if $i \leq j$, $\Gamma_{i j} \neq \emptyset$ and $\lambda_{i j}=0$. Furthermore, (13) and (14) hold by Theorem 3.11 if $i=j$.

So from now on we assume that $i<j, \Gamma_{i j} \neq \emptyset$ and $\lambda_{i j} \neq 0$. Note that $i<j$ implies that $l>1$.

Select an arbitrary vertex $u$ of $C_{i}$ and an arbitrary vertex $v$ of $C_{j}$.

Since $\lambda_{i j} \neq \mathcal{O}$ there exists at least one set $\gamma \in S_{i j}$ for which $\delta_{\gamma} \neq \emptyset$. So $\Delta_{i j} \neq \emptyset$ and $k_{i j}$ is well defined. Note that $k_{i j} \geq \# \Gamma_{i j}$. If $\gamma_{\delta}=\emptyset$ for some set $\gamma \in S_{i j}$ then there do not exist paths from $v$ to $u$ of length $n \geq \# \Gamma_{i j}$ that correspond to $\gamma$. So from now on we only consider sets $\gamma \in S_{i j}$ for which $\delta_{\gamma} \neq \emptyset$.

Let $\gamma=\left\{i_{0}, i_{1}, \ldots, i_{s}\right\} \in S_{i j}$ with $i=i_{0}<i_{1}<\ldots<i_{s}=j$. Since we assume that $\delta_{\gamma} \neq \emptyset$, we have $\hat{A}_{i_{r} i_{r}} \neq[\mathbf{0}]$ for at least one index $i_{r} \in \gamma$. Assume that $\delta_{\gamma}$ is given by $\left\{j_{0}, j_{1}, \ldots, j_{\hat{s}}\right\}$. Define

$$
\begin{array}{r}
\mathcal{S}=\left\{(U, V) \mid U=\left\{u_{0}, u_{1}, \ldots, u_{s}\right\}, V=\left\{v_{0}, v_{1}, \ldots, v_{s}\right\}, u_{s}=v, v_{0}=u,\right. \text { and } \\
\left.u_{r} \in \alpha_{i_{r}}, v_{r+1} \in \alpha_{i_{r+1}} \text { and }(\hat{A})_{u_{r} v_{r+1}} \neq \mathcal{O} \text { for } r=0,1, \ldots, s\right\} .
\end{array}
$$

Let $(U, V) \in \mathcal{S}$ with $U=\left\{u_{0}, u_{1}, \ldots, u_{s}\right\}$ and $V=\left\{v_{0}, v_{1}, \ldots, v_{s}\right\}$. Let $\mathcal{P}(\gamma, U, V)$ be the set of paths from $v$ to $u$ that pass through m.s.c.s. $C_{i_{r}}$ for $r=0,1, \ldots, s$ and that enter $C_{i_{r}}$ at vertex $u_{r}$ for $r=0,1, \ldots, s-1$ and that exit from $C_{i_{r}}$ through vertex $v_{r}$ for $r=1,2, \ldots, s$ (See also Figure 3.3). Let the sequences $\left\{\left(g_{\gamma, U, V}\right)_{k}\right\}_{k=1}^{\infty}$ and $\left\{\left(g_{\gamma}\right)_{k}\right\}_{k=1}^{\infty}$ be defined by

$$
\begin{aligned}
\left(g_{\gamma, U, V}\right)_{k} & = \begin{cases}1 & \text { if there exists a path of length } k \text { that belongs to } \mathcal{P}(\gamma, U, V) \\
\mathcal{O} & \text { otherwise }\end{cases} \\
\left(g_{\gamma}\right)_{k} & = \begin{cases}1 & \text { if there exists a path of length } k \text { that belongs to } \mathcal{P}(\gamma, U, V) \\
\mathcal{O} & \text { for some pair }(U, V) \in \mathcal{S}\end{cases}
\end{aligned}
$$

Define $c_{\gamma}=\operatorname{gcd}\left\{c_{t} \mid t \in \delta_{\gamma}\right\}$. Let us now show that the sequences $\left\{\left(g_{\gamma, U, V}\right)_{k}\right\}_{k=1}^{\infty}$ and $\left\{\left(g_{\gamma}\right)_{k}\right\}_{k=1}^{\infty}$ satisfy

$$
\left(g_{\gamma, U, V}\right)_{k+c_{\gamma}}=\left(g_{\gamma, U, V}\right)_{k} \quad \text { for all } k \geq k_{i j}
$$




$$
\begin{aligned}
\left(g_{\gamma, U, V}\right)_{k} \oplus\left(g_{\gamma, U, V}\right)_{k+1} \oplus \ldots \oplus\left(g_{\gamma, U, V}\right)_{k+c_{\gamma}-1}=1 & \text { for all } k \geq k_{i j} \\
\left(g_{\gamma}\right)_{k+c_{\gamma}}=\left(g_{\gamma}\right)_{k} & \text { for all } k \geq k_{i j} \\
\left(g_{\gamma}\right)_{k} \oplus\left(g_{\gamma}\right)_{k+1} \oplus \ldots \oplus\left(g_{\gamma}\right)_{k+c_{\gamma}-1}=1 & \text { for all } k \geq k_{i j}
\end{aligned}
$$

Note that if (16) and (17) hold for each pair $(U, V) \in \mathcal{S}$ then (18) and (19) also hold. Therefore, we now show that (16) and (17) hold. Define $\tilde{u}_{r}=u_{s}$ and $\tilde{v}_{r}=v_{s}$ if $j_{r}=i_{s}$ for $r=0,1, \ldots, \hat{s}$. We consider three cases:

Case A: $c_{j_{\tilde{r}}}=1$ for some $\tilde{r} \in\{0,1, \ldots, \hat{s}\}$.

In this case we have $c_{\gamma}=1$.

Let $l_{r}$ be the length of the shortest (possibly empty) path from vertex $\tilde{u}_{r}$ to vertex $\tilde{v}_{r}$ of $C_{j_{r}}$ for each $r \in\{0,1, \ldots, \hat{s}\}$. We have $l_{r} \leq n_{j_{r}}-1$ for each $r$. Since $\hat{A}_{j_{\tilde{r}} j_{\tilde{r}}}$ is irreducible and since $c_{j_{\tilde{r}}}=1$, it follows from Theorem 3.3 that for any integer $p \geq\left(n_{j_{\tilde{r}}}-1\right)^{2}+1$ there exists a path of length $p$ from vertex $\tilde{u}_{\tilde{r}}$ to vertex $\tilde{v}_{\tilde{r}}$ of $C_{j_{\tilde{r}}}$. If we also take into account that there are $s$ arcs of the form $v_{r+1} \rightarrow u_{r}$ for $r=0,1, \ldots, s-1$ then it follows that for any

$$
k \geq \sum_{\substack{r=0 \\ r \neq \tilde{r}}}^{\hat{s}}\left(n_{j_{r}}-1\right)+s+\left(n_{j_{\tilde{r}}}-1\right)^{2}+1 \stackrel{\text { def }}{=} K_{\mathrm{A}}
$$

there exists a path of length $k$ that belongs to $\mathcal{P}(\gamma, U, V)$. Let us now show that $K_{\mathrm{A}} \leq k_{i j}$. Let $r \in\{0,1, \ldots, \hat{s}\}$. If $c_{j_{r}}>1$ then it follows from the definition of $k_{n_{j_{r}}, c_{j_{r}}}$ that $n_{j_{r}}-1 \leq k_{n_{j_{r}}, c_{j_{r}}}$. Furthermore, if $c_{j_{r}}=1$, then we have $n_{j_{r}}-1 \leq\left(n_{j_{r}}-1\right)^{2}+1=k_{n_{j_{r}}, c_{j_{r}}}$. Since $c_{j_{\tilde{r}}}=1$, we have $\left(n_{j_{\tilde{r}}}-1\right)^{2}+1=$ $k_{n_{j_{\tilde{r}}}, c_{j_{\tilde{r}}}}$. Furthermore, $\delta_{\gamma} \subseteq \Delta_{i j}$ and $s \leq \# \Gamma_{i j}-1$. Hence, $K_{\mathrm{A}} \leq k_{i j}$, which implies that (16) and (17) hold in this case.

Case B: $c_{t}=c_{\gamma}$ and $c_{t} \neq 1$ for all $t \in \delta_{\gamma}$.

Assume that $c_{j_{\tilde{r}}}=c_{\gamma}$ with $\tilde{r} \in\{0,1, \ldots, \hat{s}\}$.

Let $l_{r}$ be the length of the shortest (possibly empty) path from vertex $\tilde{u}_{r}$ to vertex $\tilde{v}_{r}$ of $C_{j_{r}}$ for each $r \in\{0,1, \ldots, \tilde{r}-1, \tilde{r}+1, \ldots, \hat{s}\}$. So $l_{r} \leq n_{j_{r}}-1$ for each $r \neq \tilde{r}$. From Lemma 2.7 and from the proof of Theorem 3.11 it follows that there exists an integer $K_{\tilde{r}}$ with $k_{n_{j_{\tilde{r}}}, c_{j_{\tilde{r}}}} \leq K_{\tilde{r}} \leq k_{n_{j_{\tilde{r}}}, c_{j_{\tilde{r}}}}+c_{j_{\tilde{r}}}-1$ such that there exist paths of length $K_{\tilde{r}}+p c_{j_{\tilde{r}}}$ from vertex $\tilde{u}_{\tilde{r}}$ to vertex $\tilde{v}_{\tilde{r}}$ of $C_{j_{\tilde{r}}}$ for any $p \in \mathbb{N}$, while there do not exist paths of length $K_{\tilde{r}}+p c_{j_{\tilde{r}}}+q$ from $\tilde{u}_{\tilde{r}}$ to $\tilde{v}_{\tilde{r}}$ for any $p \in \mathbb{N}$ and any $q \in\left\{1,2, \ldots, c_{j \tilde{r}}-1\right\}$. So if we define

$$
K_{\mathrm{B}}=\sum_{\substack{r=0 \\ r \neq \tilde{r}}}^{\hat{s}} l_{r}+s+k_{n_{j_{\tilde{r}}}, c_{\tilde{r}}}
$$

then it follows from Lemma 2.7 that for any $k \geq K_{\mathrm{B}}$ either there exists a path of length $k+p c_{\gamma}$ that belongs to $\mathcal{P}(\gamma, U, V)$ for each $p \in \mathbb{N}$, or there do not exist paths of length $k+p c_{\gamma}$ that belong to $\mathcal{P}(\gamma, U, V)$ for any $p \in \mathbb{N}$. It is easy to verify that $K_{\mathrm{B}} \leq k_{i j}$. Hence, (16) and (17) also hold in this case.

Case C: $c_{j_{r}} \neq 1$ for all $r \in\{0,1, \ldots, \hat{s}\}$ and $c_{j_{a}} \neq c_{j_{b}}$ for some $a, b \in\{0,1, \ldots, \hat{s}\}$. From Lemma 2.7 and from the proof of Theorem 3.11 it follows that for each $r \in\{0,1, \ldots, \hat{s}\}$ there exists an integer $K_{r}$ with $k_{n_{j_{r}}, c_{j_{r}}} \leq K_{r} \leq k_{n_{j_{r}}, c_{j_{r}}}+$ $c_{j_{r}}-1$ such that there exist paths of length $K_{r}+p c_{j_{r}}$ from $\tilde{u}_{r}$ to $\tilde{v}_{r}$ for each $p \in \mathbb{N}$, while there do not exist paths of length $K_{r}+p c_{j_{r}}+q$ from $\tilde{u}_{r}$ to $\tilde{v}_{r}$ for any $p \in \mathbb{N}$ and for any $q \in\left\{1,2, \ldots, c_{j_{r}}-1\right\}$. This implies that there exist 
paths of length $K_{0}+K_{1}+\ldots+K_{\hat{s}}+s+p_{0} c_{j_{0}}+p_{1} c_{j_{1}}+\ldots+p_{\hat{s}} c_{j_{\hat{s}}}$ that belong to $\mathcal{P}(\gamma, U, V)$ for each choice of $p_{0}, p_{1}, \ldots, p_{\hat{s}}$ such that $p_{r} \geq 0$ for each $r$. Define $K_{\gamma}=\sum_{r=0}^{\hat{s}} K_{r}+s$. Since $c_{\gamma}=\operatorname{gcd}\left\{c_{t} \mid t \in \delta_{\gamma}\right\}$ there exist positive integers $w_{0}, w_{1}, \ldots, w_{\hat{s}}$ such that $c_{j_{r}}=w_{r} c_{\gamma}$ for each $r \in\{0,1, \ldots, \hat{s}\}$ and such that $\operatorname{gcd}\left(w_{0}, w_{1}, \ldots, w_{\hat{s}}\right)=1$. So for any integer $q \geq g\left(w_{0}, w_{1}, \ldots, w_{\hat{s}}\right)+1$ there exist nonnegative integers $\alpha_{0}, \alpha_{1}, \ldots, \alpha_{\hat{s}}$ such that $q=\alpha_{0} w_{0}+\alpha_{1} w_{1}+\ldots+$ $\alpha_{\hat{s}} w_{\hat{s}}$.

Since $c_{j_{a}} \neq c_{j_{b}}$ we have $w_{a} \neq w_{b}$. Therefore, we may assume without loss of generality that $w_{0}<w_{1}<\ldots<w_{\hat{s}}$ with $\hat{s} \geq 2$. We have $w_{\hat{s}}=\frac{c_{j_{\hat{s}}}}{c_{\gamma}} \leq$ $\frac{n_{j_{\hat{s}}}}{c_{\gamma}} \leq \frac{n_{r_{i j}}}{c_{\gamma}}$. Hence, $w_{0} \leq \frac{n_{r_{i j}}}{c_{\gamma}}-1$ since $w_{0}<w_{\hat{s}}$. Furthermore, $w_{0} \geq 1$ and thus $w_{\hat{s}} \geq 2$. Using a reasoning that is similar to the one used in the proof of Theorem 3.11 and Remark 3.12 we can show that

$$
g\left(w_{0}, w_{1}, \ldots, w_{\hat{s}}\right)+1 \leq\left(\frac{n_{r_{i j}}}{c_{\gamma}}\right)^{2}-3 \frac{n_{r_{i j}}}{c_{\gamma}}+2 .
$$

So if we define

$$
K_{\mathrm{C}}=K_{\gamma}+c_{\gamma}\left(\left(\frac{n_{r_{i j}}}{c_{\gamma}}\right)^{2}-3 \frac{n_{r_{i j}}}{c_{\gamma}}+2\right),
$$

then we have $K_{\mathrm{C}} \geq K_{\gamma}+c_{\gamma}\left(g\left(w_{0}, w_{1}, \ldots, w_{\hat{s}}\right)+1\right)$. Let $k \in \mathbb{N}$ with $k \geq K_{\mathrm{C}}$. Now there are two possibilities:

- If $k$ can be written as $K_{\gamma}+q c_{\gamma}$ with $q \geq g\left(w_{0}, w_{1}, \ldots, w_{\hat{s}}\right)+1$ then we have

$k=K_{\gamma}+\left(\alpha_{0} w_{0}+\alpha_{1} w_{1}+\ldots+\alpha_{\hat{s}} w_{\hat{s}}\right) c_{\gamma}=K_{\gamma}+\alpha_{0} c_{j_{0}}+\alpha_{1} c_{j_{1}}+\ldots+\alpha_{\hat{s}} c_{j_{\hat{s}}}$,

which implies that there exists a path of length $k$ that belongs to $\mathcal{P}(\gamma, U, V)$.

- On the other hand, if $k$ cannot be written as $K_{\gamma}+q c_{\gamma}$ for any $q \in \mathbb{N}$ then it follows from Lemma 2.7 that there does not exist a path of length $k$ that belongs to $\mathcal{P}(\gamma, U, V)$.

Since $k_{i j} \geq K_{\mathrm{C}}$ this implies that (16) and (17) also hold in this case.

If we consider all possible paths from vertex $v$ to vertex $u$ of length $k \in \mathbb{N}$ with $k \geq \# \Gamma_{i j}$, then each of these paths corresponds to some set $\gamma \in S_{i j}$ with $\delta_{\gamma} \neq \emptyset$. Since $\left(\hat{A}^{\otimes^{k}}\right)_{u v}$ is equal to $\mathbf{I}$ if and only if there exists a path of length $k$ from $v$ to $u$, we have

$$
\left(\hat{A}^{\otimes^{k}}\right)_{u v}=\bigoplus_{\substack{\gamma \in S_{i j} \\ \delta_{\gamma} \neq \emptyset}}\left(g_{\gamma}\right)_{k}
$$

if $k \geq \# \Gamma_{i j}$. Note that if $c_{\gamma}=1$ and $\delta_{\gamma} \neq \emptyset$ then we have $\left(g_{\gamma}\right)_{k_{i j}}=1$. Since each sequence $\left\{\left(g_{\gamma}\right)_{k}\right\}_{k=1}^{\infty}$ satisfies (18), it follows from Lemma 3.13 that

$$
\left(\hat{A}^{\otimes^{k+c_{i j}}}\right)_{u v}=\left(\hat{A}^{\otimes^{k}}\right)_{u v} \quad \text { for all } k \geq k_{i j} .
$$

Furthermore, since each sequence $\left\{\left(g_{\gamma}\right)_{k}\right\}_{k=1}^{\infty}$ satisfies (19), we have

$$
\left(\hat{A}^{\otimes^{k}} \oplus \hat{A}^{\otimes^{k+1}} \oplus \ldots \oplus \hat{A}^{\otimes^{k+c_{i j}-1}}\right)_{u v}=1 \quad \text { for all } k \geq k_{i j} .
$$

So (13) and (14) also hold if $\lambda_{i j} \neq \mathbf{0}$. 
REMARK 3.16. Note that if $\gamma_{1}, \gamma_{2} \in S_{i j}$ and $\gamma_{1} \subseteq \gamma_{2}$ then we do not have to consider $\gamma_{1}$ when we are determining $S_{i j}$. Hence, we could have defined $S_{i j}$ as the set of maximal subsets $\left\{i_{0}, i_{1}, \ldots, i_{s}\right\}$ of $\{1,2, \ldots, l\}$ with $i=i_{0}<i_{1}<\ldots<i_{s}=j$ and $\hat{A}_{i_{r} i_{r+1}} \neq \mathcal{O}$ for $r=0,1, \ldots, s-1$.

Let us now give an example in which the various sets and indices that appear in the formulation of Theorem 3.15 are illustrated.

EXAmple 3.17. Consider the matrix

$$
A=\left[\begin{array}{l|ll|lll|l}
0 & 1 & 0 & 1 & 0 & 0 & 0 \\
\hline 0 & 0 & 1 & 0 & 0 & 0 & 0 \\
0 & 1 & 0 & 0 & 0 & 0 & 1 \\
\hline 0 & 0 & 0 & 0 & 0 & 1 & 0 \\
0 & 0 & 0 & 1 & 0 & 0 & 0 \\
0 & 0 & 0 & 0 & 1 & 0 & 1 \\
\hline 0 & 0 & 0 & 0 & 0 & 0 & 0
\end{array}\right]
$$

This matrix is in Frobenius normal form and its block structure is indicated by the horizontal and vertical lines. The precedence graph of $A$ is represented in Figure 3.5. We have

$$
\begin{aligned}
& A^{\otimes^{2}}=\left[\begin{array}{l|ll|lll|l}
0 & 0 & 1 & 0 & 0 & 1 & 0 \\
\hline 0 & 1 & 0 & 0 & 0 & 0 & 1 \\
0 & 0 & 1 & 0 & 0 & 0 & 0 \\
\hline 0 & 0 & 0 & 0 & 1 & 0 & 1 \\
0 & 0 & 0 & 0 & 0 & 1 & 0 \\
0 & 0 & 0 & 1 & 0 & 0 & 0 \\
\hline 0 & 0 & 0 & 0 & 0 & 0 & 0
\end{array}\right], \quad A^{\otimes}=\left[\begin{array}{l|ll|lll|l}
0 & 1 & 0 & 0 & 1 & 0 & 1 \\
\hline 0 & 0 & 1 & 0 & 0 & 0 & 0 \\
0 & 1 & 0 & 0 & 0 & 0 & 1 \\
\hline 0 & 0 & 0 & 1 & 0 & 0 & 0 \\
0 & 0 & 0 & 0 & 1 & 0 & 1 \\
0 & 0 & 0 & 0 & 0 & 1 & 0 \\
\hline 0 & 0 & 0 & 0 & 0 & 0 & 0
\end{array}\right], \\
& A^{\otimes^{4}}=\left[\begin{array}{c|cc|ccc|c}
0 & 0 & 1 & 1 & 0 & 0 & 0 \\
\hline 0 & 1 & 0 & 0 & 0 & 0 & 1 \\
0 & 0 & 1 & 0 & 0 & 0 & 0 \\
\hline 0 & 0 & 0 & 0 & 0 & 1 & 0 \\
0 & 0 & 0 & 1 & 0 & 0 & 0 \\
0 & 0 & 0 & 0 & 1 & 0 & 1 \\
\hline 0 & 0 & 0 & 0 & 0 & 0 & 0
\end{array}\right], \quad A^{\otimes^{5}}=\left[\begin{array}{l|ll|lll|l}
0 & 1 & 0 & 0 & 0 & 1 & 1 \\
\hline 0 & 0 & 1 & 0 & 0 & 0 & 0 \\
0 & 1 & 0 & 0 & 0 & 0 & 1 \\
\hline 0 & 0 & 0 & 0 & 1 & 0 & 1 \\
0 & 0 & 0 & 0 & 0 & 1 & 0 \\
0 & 0 & 0 & 1 & 0 & 0 & 0 \\
\hline 0 & 0 & 0 & 0 & 0 & 0 & 0
\end{array}\right], \\
& A^{\otimes}=\left[\begin{array}{c|cc|ccc|c}
0 & 0 & 1 & 0 & 1 & 0 & 1 \\
\hline 0 & 1 & 0 & 0 & 0 & 0 & 1 \\
0 & 0 & 1 & 0 & 0 & 0 & 0 \\
\hline 0 & 0 & 0 & 1 & 0 & 0 & 0 \\
0 & 0 & 0 & 0 & 1 & 0 & 1 \\
0 & 0 & 0 & 0 & 0 & 1 & 0 \\
\hline 0 & 0 & 0 & 0 & 0 & 0 & 0
\end{array}\right], \quad A^{\otimes^{7}}=\left[\begin{array}{l|ll|lll|l}
0 & 1 & 0 & 1 & 0 & 0 & 1 \\
\hline 0 & 0 & 1 & 0 & 0 & 0 & 0 \\
0 & 1 & 0 & 0 & 0 & 0 & 1 \\
\hline 0 & 0 & 0 & 0 & 0 & 1 & 0 \\
0 & 0 & 0 & 1 & 0 & 0 & 0 \\
0 & 0 & 0 & 0 & 1 & 0 & 1 \\
\hline 0 & 0 & 0 & 0 & 0 & 0 & 0
\end{array}\right], \\
& A^{\otimes^{8}}=\left[\begin{array}{c|cc|ccc|c}
0 & 0 & 1 & 0 & 0 & 1 & 0 \\
\hline 0 & 1 & 0 & 0 & 0 & 0 & 1 \\
0 & 0 & 1 & 0 & 0 & 0 & 0 \\
\hline 0 & 0 & 0 & 0 & 1 & 0 & 1 \\
0 & 0 & 0 & 0 & 0 & 1 & 0 \\
0 & 0 & 0 & 1 & 0 & 0 & 0 \\
\hline 0 & 0 & 0 & 0 & 0 & 0 & 0
\end{array}\right], \quad A^{\otimes^{9}}=\left[\begin{array}{l|ll|lll|l}
0 & 1 & 0 & 0 & 1 & 0 & 1 \\
\hline 0 & 0 & 1 & 0 & 0 & 0 & 0 \\
0 & 1 & 0 & 0 & 0 & 0 & 1 \\
\hline 0 & 0 & 0 & 1 & 0 & 0 & 0 \\
0 & 0 & 0 & 0 & 1 & 0 & 1 \\
0 & 0 & 0 & 0 & 0 & 1 & 0 \\
\hline 0 & 0 & 0 & 0 & 0 & 0 & 0
\end{array}\right],
\end{aligned}
$$




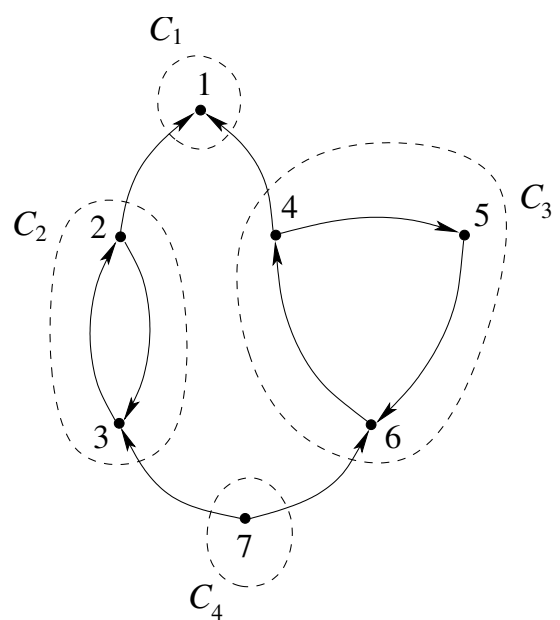

FIG. 3.5. The precedence graph $\mathcal{G}(A)$ of the matrix $A$ of Example 3.17. The subgraphs $C_{1}, C_{2}$, $C_{3}$ and $C_{4}$ are the m.s.c.s.'s of $\mathcal{G}(A)$.

$$
A^{\otimes^{10}}=\left[\begin{array}{c|cc|ccc|c}
0 & 0 & 1 & 1 & 0 & 0 & 0 \\
\hline 0 & 1 & 0 & 0 & 0 & 0 & 1 \\
0 & 0 & 1 & 0 & 0 & 0 & 0 \\
\hline 0 & 0 & 0 & 0 & 0 & 1 & 0 \\
0 & 0 & 0 & 1 & 0 & 0 & 0 \\
0 & 0 & 0 & 0 & 1 & 0 & 1 \\
\hline 0 & 0 & 0 & 0 & 0 & 0 & 0
\end{array}\right], \ldots
$$

So $A^{\otimes^{k+6}}=A^{\otimes^{k}}$ for all $k \geq 2$.

We have $\alpha_{1}=\{1\}, \alpha_{2}=\{2,3\}, \alpha_{3}=\{4,5,6\}$ and $\alpha_{4}=\{7\}$. Furthermore, $\lambda_{1}=$ $\lambda_{4}=\mathrm{O}$ and $\lambda_{2}=\lambda_{3}=\mathbf{1}$. Let us now look at the sequence $\left\{\left(A^{\otimes^{k}}\right)_{\alpha_{1} \alpha_{4}}\right\}_{k=1}^{\infty}$. We have $S_{14}=\left\{\gamma_{1}, \gamma_{2}\right\}$ with $\gamma_{1}=\{1,2,4\}$ and $\gamma_{2}=\{1,3,4\}$. So $\delta_{\gamma_{1}}=\{2\}, c_{\gamma_{1}}=2, \delta_{\gamma_{2}}=\{3\}$ and $c_{\gamma_{2}}=3$. We have $\Gamma_{14}=\{1,2,3,4\}, \Delta_{14}=\{2,3\}, \lambda_{14}=\mathbf{1}, c_{14}=\operatorname{lcm}(2,3)=6$ and $r_{14}=3$. Hence,

$$
\begin{aligned}
k_{14} & =k_{2,2}+k_{3,3}+\# \Gamma_{14}-1+\max \left(0, \frac{n_{3}^{2}}{c_{\gamma_{1}}}-3 n_{3}+2 c_{\gamma_{1}}, \frac{n_{3}^{2}}{c_{\gamma_{2}}}-3 n_{3}+2 c_{\gamma_{2}}\right) \\
& =\frac{3}{2}+4+4-1+\max \left(0, \frac{9}{2}-9+4, \frac{9}{3}-9+6\right)=\frac{17}{2} .
\end{aligned}
$$

Note that we indeed have $\left(A^{\otimes^{k+6}}\right)_{\alpha_{1} \alpha_{4}}=\left(A^{\otimes^{k}}\right)_{\alpha_{1} \alpha_{4}}$ for all $k \geq 9$. $\diamond$

LemMa 3.18. Consider $m$ positive integers $c_{1}, c_{2}, \ldots, c_{m}$. Let $c=\operatorname{lcm}\left(c_{1}, c_{2}, \ldots\right.$, $\left.c_{m}\right)$. Consider $r$ non-empty subsets $\alpha_{1}, \alpha_{2}, \ldots, \alpha_{r}$ of $\{1,2, \ldots, m\}$. Define $d_{i}=$ $\operatorname{gcd}\left\{c_{k} \mid k \in \alpha_{i}\right\}$ for each $i$. If $d=\operatorname{lcm}\left(d_{1}, d_{2}, \ldots, d_{r}\right)$, then $d$ is a divisor of $c$.

Proof. We may assume without loss of generality that $c_{i} \neq c_{j}$ for all $i, j$ with $i \neq j$. If $d$ is a divisor of $\operatorname{lcm}\left(c_{1}, c_{2}, \ldots, c_{m-1}\right)$ then it also is a divisor of $\operatorname{lcm}\left(c_{1}, c_{2}, \ldots, c_{m}\right)$. Therefore, we may assume without loss of generality that $\alpha_{1} \cup \alpha_{2} \cup \ldots \cup \alpha_{r}=$ $\{1,2, \ldots, m\}$. If $\alpha_{i} \subseteq \alpha_{j}$ then $d_{j}$ is a divisor of $d_{i}$ and then $\operatorname{lcm}\left(d_{1}, d_{2}, \ldots, d_{r}\right)=$ $\operatorname{lcm}\left(d_{1}, d_{2}, \ldots, d_{j-1}, d_{j+1}, \ldots, d_{r}\right)$, which implies that $\alpha_{j}$ is redundant and may be removed. If $d_{i}=d_{j}$ then $\alpha_{j}$ is also redundant and may be removed. It is easy to 
verify that if we remove all redundant sets, then the resulting number of sets $\alpha_{i}$ is less than or equal to $m$ (The worst cases being when $\alpha_{i}=\{1,2, \ldots, m\} \backslash\{i\}$ for $i=1,2, \ldots, m$ or when $\alpha_{i}=\{i\}$ for $i=1,2, \ldots, m$.) Hence, we may assume without loss of generality that $r \leq m$ and that $d_{i} \neq d_{j}$ for all $i, j$ with $i \neq j$.

Since $r \leq m, \alpha_{1} \cup \alpha_{2} \cup \ldots \cup \alpha_{r}=\{1,2, \ldots, m\}$ and $\alpha_{i} \not \subset \alpha_{j}$ for all $i, j$ with $i \neq j$, we can select indices $l_{1}, l_{2}, \ldots, l_{r}$ such that $l_{i} \in \alpha_{i}$ for $i=1,2, \ldots, r$ and $l_{i} \neq l_{j}$ for all $i, j$ with $i \neq j$.

Since $d_{i}=\operatorname{gcd}\left\{c_{k} \mid k \in \alpha_{i}\right\}, d_{i}$ is a divisor of $c_{l_{i}}$ for each $i$.

Since all $c_{i}$ 's are different we have

$$
c=\operatorname{lcm}\left(c_{1}, c_{2}, \ldots, c_{m}\right)=\frac{c_{1} c_{2} \ldots c_{m}}{\operatorname{gcd}\left(c_{1}, c_{2}, \ldots, c_{m}\right)} .
$$

We also have

$$
d=\frac{d_{1} d_{2} \ldots d_{r}}{\operatorname{gcd}\left(d_{1}, d_{2}, \ldots, d_{r}\right)}
$$

Since $\alpha_{1} \cup \alpha_{2} \cup \ldots \cup \alpha_{r}=\{1,2, \ldots, m\}$ we have $\operatorname{gcd}\left(c_{1}, c_{2}, \ldots, c_{m}\right)=\operatorname{gcd}\left(d_{1}, d_{2}, \ldots, d_{r}\right)$. So

$$
c=\frac{c_{1} c_{2} \ldots c_{m}}{d_{1} d_{2} \ldots d_{r}} d
$$

Since $d_{i}$ is a divisor of $c_{l_{i}}$ for $i=1,2, \ldots, r$ there exist integers $w_{i} \in \mathbb{N}$ such that $c_{l_{i}}=w_{i} d_{i}$ for $i=1,2, \ldots, r$. If we define $\beta=\{1,2, \ldots, m\} \backslash\left\{l_{1}, l_{2}, \ldots, l_{r}\right\}$, then we have

$$
c=w_{1} w_{2} \ldots w_{r}\left(\prod_{i \in \beta} c_{i}\right) d
$$

where $\prod_{i \in \emptyset} c_{i}$ is equal to 1 by definition. So $c=w d$ for some integer $w \in \mathbb{N}$, which implies that $d$ is a divisor of $c$.

Lemma 3.19. Let $n \in \mathbb{N}$ and let $c \in\{0,1, \ldots, n\}$. If $k_{n, c}$ is defined by (7) if $c>0$ and if $k_{n, c}=0$ if $c=0$, then we have $k_{n, c} \leq(n-1)^{2}+1$.

Proof. It is obvious that the lemma holds if $c=0$ or if $c=1$. So from now on we assume that $c>1$.

Define $f(c)=\frac{n^{2}-1}{2}+\frac{n^{2}}{c}-3 n+2 c$. Let us now show that $f(c) \leq(n-1)^{2}+1$.

We have $\frac{d f}{d c}=-\frac{n^{2}}{c^{2}}+2$. So $f$ reaches a local minimum in $c=\frac{n}{\sqrt{2}}$ and is decreasing if $1<c<\frac{n}{\sqrt{2}}$ and increasing if $c>\frac{n}{\sqrt{2}}$.

Let us first consider the cases where $n$ is equal to 2 or to 3 . If $n=2$ then we have $c=2$ and $f(c)=\frac{4-1}{2}+2-6+4=\frac{3}{2} \leq 2=(2-1)^{2}+1$. If $n=3$ then $\frac{n}{\sqrt{2}}$ does not belong to the interval $[2,3]$ and then the maximal value of $f$ in $[2,3]$ is equal to $f(3)=\frac{9-1}{2}+\frac{9}{3}-9+6=\frac{9}{2} \leq 5=(3-1)^{2}+1$.

From now on we assume that $n$ is larger than or equal to 4 . If $n \geq 4$, then $\frac{n}{\sqrt{2}}$ belongs to the interval $[2, n]$ and then the maximal value of $f$ in $[2, n]$ is equal to

$$
\begin{aligned}
\max (f(2), f(n)) & =\max \left(\frac{n^{2}-1}{2}+\frac{n^{2}}{2}-3 n+4, \frac{n^{2}-1}{2}+n-3 n+2 n\right) \\
& =\max \left(n^{2}-3 n+\frac{7}{2}, \frac{n^{2}}{2}-\frac{1}{2}\right)
\end{aligned}
$$




$$
\begin{array}{ll}
=n^{2}-3 n+\frac{7}{2} & (\text { since } n \geq 4) \\
\leq(n-1)^{2}+1 & (\text { since } n \geq 2) .
\end{array}
$$

Hence, $k_{n, c} \leq(n-1)^{2}+1$.

THEOREM 3.20. Let $A \in \mathbb{B}^{n \times n}$ and let $c$ be the cyclicity of $A$. We have $A^{\otimes^{k+c}}=$ $A^{\otimes^{k}}$ for all $k \geq 2 n^{2}-3 n+2$.

Proof. Let $\hat{A}=P \otimes A \otimes P^{T}$ be the Frobenius normal from of $A$ where $P$ is a permutation matrix. Assume that $\hat{A}$ is a matrix of the form (1) with $\hat{A}_{i i}$ irreducible for $i=1,2, \ldots, l$. Consider $i, j \in\{1,2, \ldots, l\}$ with $i \leq j$. Let $S_{i j}, \Gamma_{i j}, \Delta_{i j}, \lambda_{i j}, c_{i j}$, $r_{i j}$ and $k_{i j}$ be defined as in Theorem 3.15. Let us now show that $k_{i j} \leq 2(n-1)^{2}+n$. It is easy to verify that this holds if $i=j$, or if $i<j$ and $\lambda_{i j}=0$. So from now on we assume that $\lambda_{i j} \neq \mathcal{O}$. Hence,

$$
k_{i j}=\sum_{t \in \Delta_{i j}} k_{n_{t}, c_{t}}+\# \Gamma_{i j}-1+\max \left(0 \max _{\substack{\gamma \in S_{i j} \\ \delta_{\gamma} \neq \emptyset}}\left\{\frac{n_{r_{i j}}^{2}}{c_{\gamma}}-3 n_{r_{i j}}+2 c_{\gamma}\right\}\right) .
$$

Since we have $k_{n_{i}, c_{i}} \leq\left(n_{i}-1\right)^{2}+1$ for each $i \in\{1,2, \ldots, l\}$ by Lemma 3.19, it follows from the proof of Lemma 3.8 that

$$
\sum_{t \in \Delta_{i j}} k_{n_{t}, c_{t}} \leq(n-1)^{2}+1
$$

Furthermore, $\# \Gamma_{i j} \leq j-i+1 \leq n$.

Let us now show that

$$
\frac{n_{r_{i j}}^{2}}{c_{\gamma}}-3 n_{r_{i j}}+2 c_{\gamma} \leq(n-1)^{2}
$$

for each $\gamma \in S_{i j}$ with $\delta_{\gamma} \neq \emptyset$. Let $\gamma \in S_{i j}$ with $\delta_{\gamma} \neq \emptyset$. From Lemma 2.6 it follows that $c_{t} \leq n_{t}$ for each $t \in \delta_{\gamma}$. Hence, $1 \leq c_{\gamma}=\operatorname{gcd}\left\{n_{t} \mid t \in \delta_{\gamma}\right\} \leq \max \left\{n_{t} \mid t \in \delta_{\gamma}\right\} \leq n_{r_{i j}}$. Define $f(c)=\frac{n_{r_{i j}}^{2}}{c_{n}}-3 n_{r_{i j}}+2 c$. From the proof of Lemma 3.19 it follows that $f$ is decreasing if $c<\frac{n_{r_{i j}}}{\sqrt{2}}$ and increasing if $c>\frac{n_{r_{i j}}}{\sqrt{2}}$.

If $n_{r_{i j}}=1$ then we have $c_{\gamma}=1$ and $f\left(c_{\gamma}\right)=1-3+2=0 \leq 0=(1-1)^{2}$.

If $n_{r_{i j}}>1$ then the maximum value of $f$ in the interval $\left[1, n_{r_{i j}}\right]$ is equal to

$$
\begin{aligned}
\max \left(f(1), f\left(n_{r_{i j}}\right)\right) & =\max \left(n_{r_{i j}}^{2}-3 n_{r_{i j}}+2, n_{r_{i j}}-3 n_{r_{i j}}+2 n_{r_{i j}}\right) \\
& =\max \left(\left(n_{r_{i j}}-1\right)^{2}+1-n_{r_{i j}}, 0\right) \\
& \leq\left(n_{r_{i j}}-1\right)^{2} \quad\left(\text { since } n_{r_{i j}} \geq 1\right) \\
& \leq(n-1)^{2} .
\end{aligned}
$$

Hence, $k_{i j} \leq 2(n-1)^{2}+n=2 n^{2}-3 n+2$. Furthermore, $c_{i j}$ is a divisor of $c$ by Lemma 3.18. Hence, $\hat{A}^{\otimes^{k+c}}=\hat{A}^{\otimes^{k}}$ for all $k \geq 2 n^{2}-3 n+2$. Since, $A^{\otimes^{k}}=P^{T} \otimes \hat{A}^{\otimes^{k}} \otimes P$ for all $k \in \mathbb{N}$, this implies that $A^{\otimes^{k+c}}=A^{\otimes^{k}}$ for all $k \geq 2 n^{2}-3 n+2$.

\section{Applications and extensions.}


4.1. Markov chains. It is often possible to represent the behavior of a physical system by describing all the different states the system can occupy and by specifying how the system moves from one state to another at each time step. If the state space of the system is discrete and if the future evolution of the system only depends on the current state of the system and not on past history, the system may be represented by a Markov chain. Markov chains can be used to describe a wide variety of systems and phenomena in domains such as diffusion processes, genetics, learning theory, sociology, economics, and so on [22].

A finite homogeneous Markov chain is a stochastic process with a finite number of states $s_{1}, s_{2}, \ldots, s_{n}$ where the transition probability to go from one state to another state only depends on the current state and is independent of the time step. We define an $n$ by $n$ matrix $P$ such that $p_{i j}$ is equal to the probability that the next state is $s_{i}$ given that the current state is $s_{j}$. Note that $p_{i j} \geq 0$ for all $i, j$. We define a sequence of vectors $\{\theta(k)\}_{k=0}^{\infty}$ with $\theta(k) \in[0,1]^{n}$ where $(\theta(k))_{i}$ is the probability that the system is in state $s_{i}$ at time step $k$. If the initial probability vector $\theta(0)$ is given, the evolution of the system is described by

$$
\theta(k+1)=P \theta(k) \quad \text { for } k \in \mathbb{N} .
$$

Hence,

$$
\theta(k)=P^{k} \theta(0) \quad \text { for } k \in \mathbb{N} \text {. }
$$

So if we consider the Boolean algebra $(\{0, \mathfrak{p}\},+, \cdot)$ where $\mathfrak{p}$ stands for an arbitrary positive number and if we define a matrix $\tilde{P} \in\{0, \mathfrak{p}\}^{n \times n}$ such that $\tilde{p}_{i j}=0$ if $p_{i j}=0$ and $\tilde{p}_{i j}=\mathfrak{p}$ if $p_{i j}>0$, then we can give the following interpretation to the Boolean matrix power $\tilde{P}^{\otimes^{k}}$. We can go from state $s_{j}$ to state $s_{i}$ in $k$ steps if and only if $\left(P^{k}\right)_{i j}>0$ or equivalently if $\left(\tilde{P}^{\otimes^{k}}\right)_{i j}=\mathfrak{p}$. As a consequence, the results of this paper can also be used to obtain upper bounds for the length of the transient behavior of a finite homogeneous Markov chain.

For more information on Markov chains and their applications the interested reader is referred to $[2,12,20,22]$ and the references therein.

4.2. Max-plus algebra. Our main motivation for studying sequences of consecutive powers of a matrix in a Boolean algebra lies in the max-plus-algebraic system theory for discrete event systems. Typical examples of discrete event systems are flexible manufacturing systems, telecommunication networks, parallel processing systems, traffic control systems and logistic systems. The class of the discrete event systems essentially consists of man-made systems that contain a finite number of resources (e.g., machines, communications channels or processors) that are shared by several users (e.g., product types, information packets or jobs) all of which contribute to the achievement of some common goal (e.g., the assembly of products, the end-to-end transmission of a set of information packets, or a parallel computation).

There are many modeling and analysis techniques for discrete event systems, such as queuing theory, (extended) state machines, max-plus algebra, formal languages, automata, temporal logic, generalized semi-Markov processes, Petri nets, perturbation analysis, computer simulation and so on (See $[1,6,19,23,24]$ and the references cited therein). In general models that describe the behavior of a discrete event system are nonlinear in conventional algebra. However, there is a class of discrete event systems - the max-linear discrete event systems - that can be described by a model that is 
"linear" in the max-plus algebra $[1,7,8]$. The model of a max-linear discrete event system can be characterized by a triple of matrices $(A, B, C)$, which are called the system matrices of the model.

One of the open problems in the max-plus-algebraic system theory is the minimal realization problem, which consists in determining the system matrices of the model of a max-linear discrete event system starting from its "impulse response" such that the dimensions of the system matrices are as small as possible (See [1] for more information). In order to tackle the general minimal realization problem it is useful to first study a simplified version: the Boolean minimal realization problem, in which only models with Boolean system matrices are considered. The results of this paper on the length of the transient part of the sequence of consecutive powers of a matrix in a Boolean algebra can be used to obtain some results for the Boolean minimal realization problem in the max-plus-algebraic system theory for discrete event systems [10]: they can be used to obtain a lower bound for the minimal system order (i.e., the smallest possible size of the system matrix $A$ ) and to prove that the Boolean minimal realization problem in the max-plus algebra is decidable (and can be solved in a time that is bounded from above by a function that is exponential in the minimal system order).

Both Boolean algebras and the max-plus algebra are special cases of a dioid (i.e., an idempotent semiring) $[1,16]$. For applications of dioids in graph theory, generating languages and automata theory the interested reader is referred to $[14,15,16]$.

4.3. Extensions. In this paper we have restricted ourselves to Boolean algebras. In this section we give some examples that illustrate some of the phenomena that could occur when we want to extend our results to more general algebraic structures. In our examples we shall use the max-plus algebra $(\mathbb{R} \cup\{-\infty\}$, max, +$)$, but for other extensions of Boolean algebras similar examples can be constructed.

In contrast to Boolean algebra (cf. Theorem 3.20) the sequence of consecutive powers of a matrix in a more general algebraic structure does not always reach a stationary or cyclic regime after a finite number of terms as is shown by the following example.

EXAmPle 4.1. Consider the matrix

$$
A=\left[\begin{array}{rr}
0 & -\infty \\
-\infty & -1
\end{array}\right]
$$

Since the $k$ th max-plus-algebraic power of $A$ is given by

$$
A^{\otimes^{k}}=\left[\begin{array}{rr}
0 & -\infty \\
-\infty & -k
\end{array}\right]
$$

for $k \in \mathbb{N}_{0}$, the sequence $\left\{A^{\otimes^{k}}\right\}_{k=1}^{\infty}$ does not reach a stationary or cyclic regime in a finite number of steps.

Note that the matrix $A$ of Example 4.1 is not irreducible. However, if a matrix is irreducible then it can be shown $[1,7,13]$ that the sequence of consecutive max-plusalgebraic powers of the given matrix always reaches a cyclic regime of the form (2) after a finite number of terms. However, even if the sequence of consecutive powers reaches a stationary regime then in general the length of the transient part will not

\footnotetext{
${ }^{4}$ This is the output of the system when a certain standardized input sequence is applied to the system.
} 
only depend on the size and the cyclicity of the matrix but also on the range and the resolution (i.e., on the size of the representation) of the finite elements of the matrix as is shown by the following examples.

EXAmple 4.2. Let $N \in \mathbb{N}$ and consider

$$
A(N)=\left[\begin{array}{rr}
-1 & -N \\
0 & 0
\end{array}\right] .
$$

The matrix $A(N)$ is irreducible and has cyclicity 1 and its $\lambda$-value ${ }^{5}$ is equal to 0 . The $k$ th max-plus-algebraic power of $A(N)$ is given by

$$
(A(N))^{\otimes^{k}}=\left[\begin{array}{rr}
\max (-k,-N) & -N \\
0 & 0
\end{array}\right]
$$

for each $k \in \mathbb{N}_{0}$. This implies that the smallest integer $k_{0}$ for which (2) holds, is given by $k_{0}=N$, i.e., $k_{0}$ depends on the range of the finite entries of $A(N)$.

EXAMPLE 4.3. Let $\varepsilon>0$ and consider the matrix

$$
A(\varepsilon)=\left[\begin{array}{rr}
0 & 0 \\
-1 & -\varepsilon
\end{array}\right]
$$

This matrix is irreducible, has cyclicity 1 and its $\lambda$-value is equal to 0 . Since the $k$ th max-plus-algebraic power of $A(\varepsilon)$ is given by

$$
(A(\varepsilon))^{\otimes^{k}}=\left[\begin{array}{rr}
0 & 0 \\
-1 & \max (-1,-k \varepsilon)
\end{array}\right],
$$

the smallest integer $k_{0}$ for which (2) holds, is $k_{0}=\left\lceil\frac{1}{\varepsilon}\right\rceil$. So this example - which has been inspired by the example on p. 152 of [1] — shows that in general the length of the transient part of the sequence $\left\{A^{\otimes^{k}}\right\}_{k=1}^{\infty}$ depends on the resolution of the finite entries of $A$.

5. Conclusions. In this paper we have studied the ultimate behavior of the sequence of consecutive powers of a matrix in a Boolean algebra, and we have derived some upper bounds for the length of the transient part of this sequence. The results that have been derived in this paper can be used in the analysis of the transient behavior of Markov chains and in the max-plus-algebraic system theory for discrete event systems.

Topics for future research are the derivation of tighter upper bounds for the length of the transient part of the sequence of consecutive power of a matrix in a Boolean algebra, and extension of our results to more general algebraic structures such as the max-plus algebra.

Acknowledgments. The authors want to thank the anonymous reviewers for their useful comments and remarks, and for pointing out the connection with Markov chains.

Bart De Moor is a research associate with the F.W.O. (Fund for Scientific Research - Flanders). This research was sponsored by the Concerted Action Project of the Flemish Community, entitled "Model-based Information Processing Systems" (GOAMIPS), by the Belgian program on interuniversity attraction poles (IUAP P4-02 and IUAP P4-24), by the ALAPEDES project of the European Community Training and Mobility of Researchers Program.

\footnotetext{
${ }^{5}$ For methods to compute the number $\lambda$ that appears in Theorem 2.5 for a max-plus-algebraic matrix the reader is referred to $[1,3,7,21]$.
} 


\section{REFERENCES}

[1] F. Baccelli, G. Cohen, G. Olsder, And J. Quadrat, Synchronization and Linearity, John Wiley \& Sons, New York, 1992.

[2] A. Berman and R. Plemmons, Nonnegative Matrices in the Mathematical Sciences, Academic Press, New York, 1979.

[3] J. BRAKer And G. Olsder, The power algorithm in max algebra, Linear Algebra and Its Applications, 182 (1993), pp. 67-89.

[4] A. Brauer, On a problem of partitions, American Journal of Mathematics, 64 (1942), pp. 299312 .

[5] R. Brualdi And H. Ryser, Combinatorial Matrix Theory, vol. 39 of Encyclopedia of Mathematics and Its Applications, Cambridge University Press, Cambridge, UK, 1991.

[6] C. Cassandras, S. Lafortune, And G. Olsder, Introduction to the modelling, control and optimization of discrete event systems, in Trends in Control: A European Perspective, A. Isidori, ed., Springer-Verlag, Berlin, Germany, 1995, pp. 217-291.

[7] G. Cohen, D. Dubois, J. Quadrat, and M. Viot, A linear-system-theoretic view of discreteevent processes and its use for performance evaluation in manufacturing, IEEE Transactions on Automatic Control, 30 (1985), pp. 210-220.

[8] R. Cuninghame-Green, Minimax Algebra, vol. 166 of Lecture Notes in Economics and Mathematical Systems, Springer-Verlag, Berlin, Germany, 1979.

[9] B. De Schutter, Max-Algebraic System Theory for Discrete Event Systems, PhD thesis, Faculty of Applied Sciences, K.U.Leuven, Leuven, Belgium, Feb. 1996.

[10] B. De Schutter, V. Blondel, R. De Vries, And B. De Moor, On the boolean minimal realization problem in the max-plus algebra, Tech. Rep. 97-68, ESAT-SISTA, K.U.Leuven, Leuven, Belgium, Dec. 1997. Accepted for publication in Systems \& Control Letters.

[11] P. Erdös and R. Graham, On a linear Diophantine problem of Frobenius, Acta Arithmetica, 21 (1972), pp. 399-408.

[12] F. Gantmacher, The Theory of Matrices, vol. 2, Chelsea Publishing Company, New York, 1959.

[13] S. GauberT, On rational series in one variable over certain dioids, Tech. Rep. 2162, INRIA, Le Chesnay, France, Jan. 1994.

[14] M. Gondran And M. Minoux, Eigenvalues and eigenvectors in semimodules and their interpretation in graph theory, in Proceedings of the 9th International Mathematical Programming Symposium, Budapest, Hungary, Aug. 1976, pp. 333-348.

[15] —, Graphs and Algorithms, John Wiley \& Sons, Chichester, 1984.

[16] - Linear algebra in dioids: A survey of recent results, Annals of Discrete Mathematics, 19 (1984), pp. 147-163.

[17] F. HARARY, A graph theoretic approach to matrix inversion by partitioning, Numerische Mathematik, 4 (1962), pp. 128-135.

[18] - Graph Theory, Addison-Wesley, Reading, Massachusetts, 1969.

[19] Y. Ho, ed., Discrete Event Dynamic Systems: Analyzing Complexity and Performance in the Modern World, IEEE Press, Piscataway, New Jersey, 1992.

[20] D. IsaAcson And R. Madsen, Markov Chains, Theory and Applications, John Wiley \& Sons, New York, 1976.

[21] R. KARP, A characterization of the minimum cycle mean in a digraph, Discrete Mathematics, 23 (1978), pp. 309-311.

[22] J. Kemeny, J. Snell, And A. Knapp, Denumerable Markov Chains, Springer-Verlag, New York, 1976. Reprint of the 1960 edition published by Van Nostrand, Princeton, New Jersey, in the University Series in Undergraduate Mathematics.

[23] J. Peterson, Petri Net Theory and the Modeling of Systems, Prentice-Hall, Englewood Cliffs, New Jersey, 1981.

[24] Proceedings of the IEEE, 77 (1989). Special issue on Dynamics of Discrete Event Systems.

[25] R. VArga, Matrix Iterative Analysis, Prentice-Hall, Englewood Cliffs, New Jersey, 1962.

[26] M. WANG, Y. LI, AND H. LIU, On periodicity analysis and eigen-problem of matrix in maxalgebra, in Proceedings of the 1991 IFAC Workshop on Discrete Event System Theory and Applications in Manufacturing and Social Phenomena, Shenyang, China, June 1991, pp. $44-48$.

[27] R. WiLson, Introduction to graph theory, Longman Group, London, 2nd ed., 1979. 